\title{
Analysis of the Effect of Clock Drifts on Frequency Regulation and Power Sharing in Inverter-based Islanded Microgrids
}

\author{
Pau Martí, Javier Torres-Martínez, Carlos Xavier Rosero, Manel Velasco, Jaume Miret, Member, IEEE, \\ and Miguel Castilla
}

\begin{abstract}
Local hardware clocks in physically distributed computation devices hardly ever agree because clocks drift apart and the drift can be different for each device. This paper analyses the effect that local clock drifts have in the parallel operation of voltage source inverters (VSIs) in islanded microgrids (MG). The state-of-the-art control policies for frequency regulation and active power sharing in VSIs-based MGs are reviewed and selected prototype policies are then re-formulated in terms of clock drifts. Next, steady-state properties for these policies are analyzed. For each of the policies, analytical expressions are developed to provide an exact quantification of the impact that drifts have on frequency and active power equilibrium points. In addition, a closed-loop model that accommodates all the policies is derived, and the stability of the equilibrium points is characterized in terms of the clock drifts. Finally, the implementation of the analyzed policies in a laboratory MG provides experimental results that confirm the theoretical analysis.
\end{abstract}

Index Terms-Microgrids, islanded mode, distributed control, frequency regulation, active power sharing, clock drifts, performance evaluation

\section{INTRODUCTION}

Computation devices are equipped with hardware clocks which run at a not necessarily identical speed. As a consequence, clocks of distributed devices may differ [1]. This situation leads to inaccurate and inefficient performance of many applications and protocols in distributed systems [2].

Microgrids (MGs), that are composed by distributed generation units, loads and storage systems [3] operated with digital computation and communication technology [4]-[7], may also suffer from drifting clocks. This paper investigates the effect of local clock drifts on the parallel operation of voltage source inverters (VSIs) in islanded MGs, with emphasis on frequency regulation and active power sharing. The accomplishment of these two control goals can be achieved following different strategies [8]-[10]. Among them, several approaches follow a standard hierarchical control architecture [11], [12] where primary control applies the well-known droop method [13][15] to achieve active power sharing while secondary control (in different forms, see e.g. [16]-[24]) corrects the steady-state

P. Martí, C. X. Rosero, and M. Velasco are with the Automatic Control Department, Technical University of Catalonia, Pau Gargallo 5, 08028 Barcelona, Spain, email: \{pau.marti,carlos.xavier.rosero,manel.velasco\}@upc.edu

J. Torres-Marínez, J. Miret and M. Castilla are with the Electronic Engineering Department, Technical University of Catalonia, Av. Victor Balaguer s/n, 08800 Vilanova i la Geltrú, Spain, email: \{jaume.mire,miquel.castillat\}@upc.edu, jtm90.35@gmail.com frequency deviation introduced by the droop using an integrallike control. Alternatively, recent approaches have considered different strategies where the hierarchical approach is avoided, e.g. [25], [26].

The impact of clocks drifts in frequency control and power sharing in MG has been recently investigated in [27]-[32] . The work in [27] shows that droop-controlled VSI-based MG are robust to clock drifts, although small steady-state deviations in active power sharing are observed. An extension providing a deeper modeling, analysis and experimental validation is presented in [29]. In [28] it is shown that local clock drifts in local integral controllers designed for frequency regulation make the MG unstable, results that are illustrated with simulations. The work by [30] provides an steady-state analysis of several distributed secondary frequency control strategies with consideration to clock drifts, illustrated with simulation results. In [31], and focusing in angle droop control, stabilizing controllers based in consensus are derived to remove the negative effects that drifts have on frequency control and power sharing. Simulation results are also used to corroborate the control proposal. Finally, [32] analyses the impact of clock drifts on local secondary control schemes that do not exchange control data.

The contribution of this paper is to provide a theoretical and experimental analysis of state-of-the-art control policies for parallel operation of VSIs in islanded MG. The analysis focuses on steady-state properties of the frequency control and active power sharing, as well as on stability properties considering clock drifts. It covers the results presented in [27][30], and extends them with additional policies, with steadystate expressions of the equilibrium points, with a robust stability characterization of the equilibrium points, and/or with experimental validation. In addition, it complements the analysis of local policies given in [32] because the focus of this paper is mainly on control schemes that exchange control data to accomplish the frequency control. Note however that the specific effect that communications have in the performance of the analyzed polices has not characterized. The performance evaluation paper [33] focuses on this topic.

The paper is structured as follows. The problem formulation is given in Section II. A set of representative prototype policies for frequency restoration and active power sharing are selected in Section III. The MG steady-steady state equilibrium points in the presence of drifts are analyzed in Section IV and analytical expressions that relate the steady-state frequency 
and active power of each policy as a function of the VSI local clock drifts are provided. Section V presents the closed-loop modeling approach and stability analysis considering clock drifts. Experimental results on a laboratory MG reported in Section VI illustrate the operation of the reviewed policies, corroborating the theoretical analysis. Conclusions are summarized in Section VII.

\section{PROBlem Formulation}

The MG system considered in this paper is a power network that gathers distributed generation (DG) units, inverters, loads and storage elements that can operate in grid-connected or islanded mode. The analysis of MG system can be made using the standard power flow equations for $n$ inverters given by [34]

$$
\begin{aligned}
P_{i}(t)= & G_{i i} V_{i}^{* 2}(t)-\sum_{j=1}^{n}\left(G_{i j} \cos \left(\Phi_{i}(t)-\Phi_{j}(t)\right)\right. \\
& \left.+B_{i j} \sin \left(\Phi_{i}(t)-\Phi_{j}(t)\right)\right) V_{j}^{*}(t) V_{i}^{*}(t) \\
Q_{i}(t)=- & B_{i i} V_{i}^{* 2}(t)-\sum_{j=1}^{n}\left(B_{i j} \cos \left(\Phi_{i}(t)-\Phi_{j}(t)\right)\right. \\
- & \left.G_{i j} \sin \left(\Phi_{i}(t)-\Phi_{j}(t)\right)\right) V_{j}^{*}(t) V_{i}^{*}(t)
\end{aligned}
$$

where $P_{i}(t)$ and $Q_{i}(t)$ are the active and reactive power injections of the $i^{\text {th }}$ inverter, $G_{i i}$ and $B_{i i}$ are the real and imaginary parts of the local admittance, $G_{i j}$ and $B_{i j}$ are the real and imaginary parts of the line admittance, $V_{i}^{*}(t)$ is the inverter computed set-point voltage amplitude, and $\Phi_{i}$ is the inverter voltage phase given by

$$
\Phi_{i}(t)=\int \omega_{i}^{*}(t) \mathrm{d} t
$$

where $\omega_{i}^{*}(t)$ is each inverter computed set-point voltage frequency. Figure 1 provides a conceptual block diagram of the MG where the plant, named $H$, is modeled using (1)(2), having as inputs the set of set-point frequencies and amplitudes, $\omega_{i}^{*}(t)$ and $V_{i}^{*}(t)$ respectively, and the active and reactive power as outputs, $P_{i}$ and $Q_{i}$ respectively. Henceforth, the paper focuses on frequency regulation and active power sharing in islanded MGs while voltage amplitude and reactive power control is deliberately omitted because no standard policy has been recognized to be the best and required practice, as it can be concluded from [10].

Figure 2 illustrates the type of $\mathrm{MG}$ adopted in this paper, highlighting the main parts being considered like buses (thick lines), interconnection between them (thin lines), loads (arrows) and inverter-based DGs (dashed boxes). The figure highlights the fact that each inverter, and more specifically, each micro-processor is driven by a local clock.

In each $i^{t h}$ inverter, $\omega_{i}^{*}(t)$ is calculated by the microprocessor with local measures (and possibly data received from other VSIs) in local time coordinates $t_{i}$ given by local clocks. Let the time progression at each VSI be defined as a function of the global time. Specifically, let $t_{i} \in \mathbb{R}^{+}$denote the local times at each inverter and let $t \in \mathbb{R}^{+}$denote the global time. Hence, VSIs local times can be described by

$$
t_{i}=d_{i} t
$$

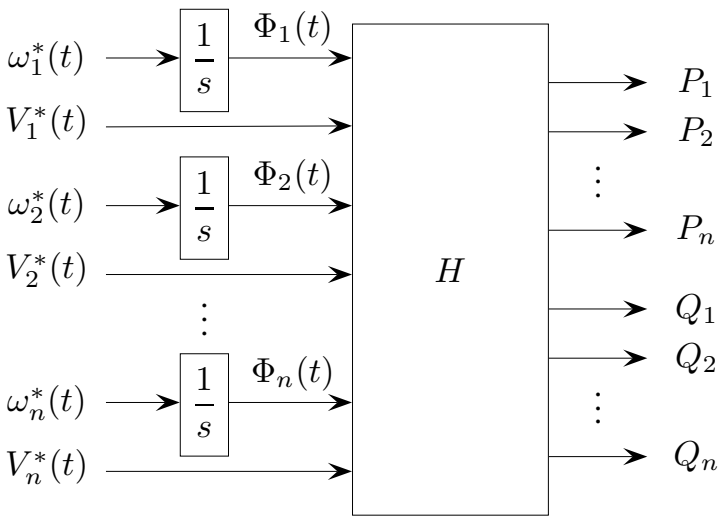

Fig. 1: Block diagram of the plant

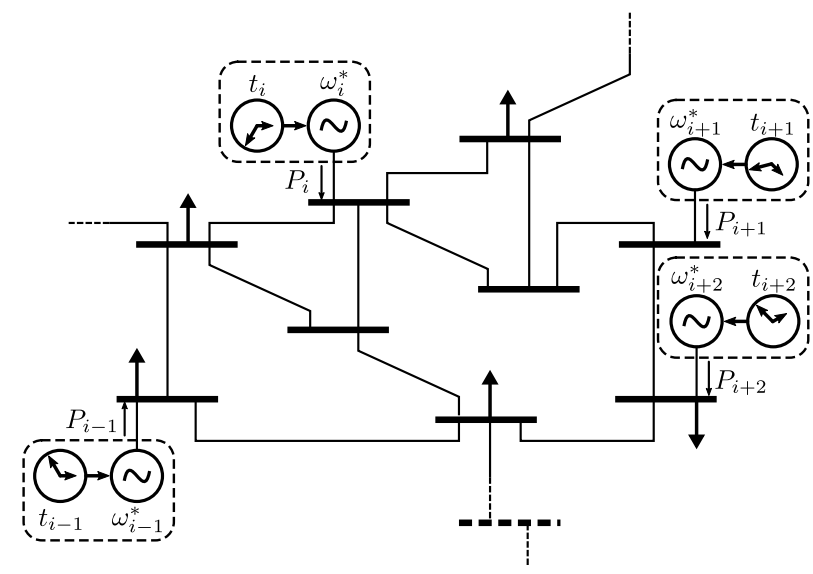

Fig. 2: Scheme of the inverter-based islanded microgrid

where $d_{i}$ is the drift rate of the $i^{t h}$ VSI local clock and it denotes the rate (frequency) of the clock. Note that in the local time definition (4), the clock offset is explicitly omitted. The offset is the difference in value from local time $t_{i}$ to global time $t$. This is done on purpose because although not all clocks are initially synchronized, i.e. $t_{0, i}$ is not the same for all VSI, local clocks are occasionally synchronized due to the phase-locked loop connection of each VSI.

In the absence of global time, the set-point frequencies $\omega_{i}^{*}(t)$ generated by the digital controllers differ between them [29]. Applying different set-point frequencies to the standard power flow equation of the active power (1) it can be observed that the $P_{i}$ steady-state values deviate. Consider the standard approximation that assumes that when phases $\Phi_{i}(t)$ are similar, then

$$
\left\{\begin{array}{l}
\cos \left(\Phi_{i}(t)-\Phi_{j}(t)\right) \simeq \cos (0)=1 \\
\sin \left(\Phi_{i}(t)-\Phi_{j}(t)\right) \simeq \Phi_{i}(t)-\Phi_{j}(t)
\end{array}\right.
$$

Then the active power (1) (and considering (3)) evolves to

$$
\begin{aligned}
P_{i}(t)= & G_{i i} V_{i}^{* 2}(t)-\sum_{j=1}^{n}\left(G_{i j}\right. \\
& \left.+B_{i j} \int \omega_{i}^{*}(t)-\omega_{j}^{*}(t) \mathrm{d} t\right) V_{j}^{*}(t) V_{i}^{*}(t)
\end{aligned}
$$

That is, $P_{i}$ values at each inverter will not settle if the setpoint frequencies $\omega_{i}^{*}(t)$ and $\omega_{j}^{*}(t)$ are different. The integral 
will increase (or decrease) infinitely, that is, no equilibrium point exists due to local clock drifts in open-loop operation.

The problem to be solved it to analyze if existing control strategies for frequency regulation and active power sharing are able to cope with this undesired unstable scenario and quantify their performance as a function of clock drifts.

\section{FREQUENCY CONTROL AND POWER SHARING}

\section{A. Selected policies}

The most common approach to the regulation of voltage frequency and amplitude in the parallel operation of VSIs is achieved by computing the set-point frequency $\omega_{i}^{*}(t)$ and amplitude $V^{*}(t)$ for the inverter current and voltage internal control loops. For power sharing, the droop method [13] is commonly applied, which is given by

$$
\begin{aligned}
\omega_{i}^{*}(t) & =\omega_{0}-m_{p} P_{i}(t) \\
V_{i}^{*}(t) & =V_{0}-n_{q} Q_{i}(t)
\end{aligned}
$$

where $\omega_{0}$ and $V_{0}$ are the nominal voltage frequency and amplitude, $P_{i}(t)$ and $Q_{i}(t)$ are the inverter output active and reactive power, $m_{p}$ and $n_{q}$ are the proportional control gains, and equation (6) is called frequency droop control and equation (7) is called voltage droop control. In the standard definition of droop, which corresponds to (6) and (7), control actions are done relative to a desired active and reactive power set-point, $P_{0 i}$ and $Q_{0 i}$ respectively [13], which is determined by long-term objectives (e.g. tertiary control). In (6)-(7) these set-points have been omitted but their inclusion does not alter the paper results. In addition, the measured rather than the normalized values for active and reactive power are used because working with dimensionless values could hide the real impact of clock drifts. Hence, without loosing generality, all inverters are assumed to have the same nominal power. Both droop methods (6) and (7) introduce deviations in the frequency and amplitude, to be corrected by the secondary control. Since the paper restricts the focus on frequency regulation and active power sharing, the considered droop control is (6). It will be further complemented with a corrective term for frequency restoration, thus leading to different secondary control policies. The standard voltage droop control (7) will not be further complemented. By fixing this policy, a common comparison framework is stablished for performance evaluation.

The analyzed policies that apply together with the droop method (6), namely droop-based policies, will be based on

$$
\omega_{i}^{*}(t)=\omega_{0}-m_{p} P_{i}(t)+\delta_{i}(t)
$$

where $\delta_{i}(t)$ is a corrective term that operates as an integral-like control of the frequency error, and it determines the features of the complete control scheme. For completeness, the analysis also considers a recent alternative approach for frequency regulation and power sharing named droop-free [25], where the nominal frequency is modified by a proportional function of the inverter output active power $P_{i}(t)$ and the rest of $n-1 \mathrm{MG}$ inverters active power $P_{j}(t)$, provided that communications are available. It has the form

$$
\omega_{i}^{*}(t)=\omega_{0}+m_{p}^{\prime} \sum_{j=1}^{n} b_{i, j}\left(P_{j}(t)-P_{i}(t)\right)
$$

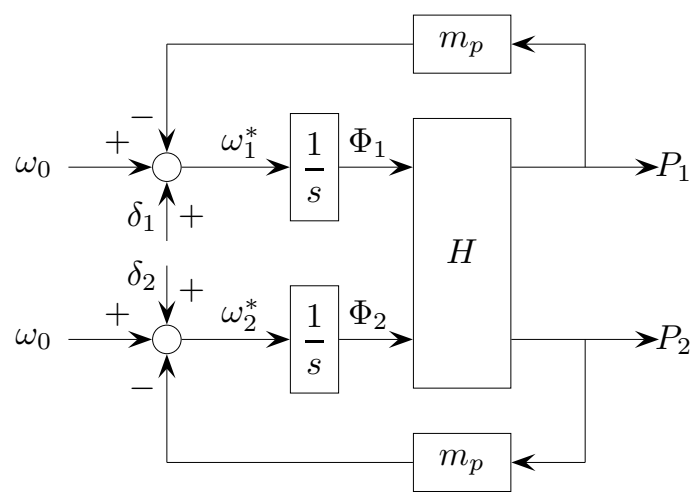

(a) Droop-based policies (8)

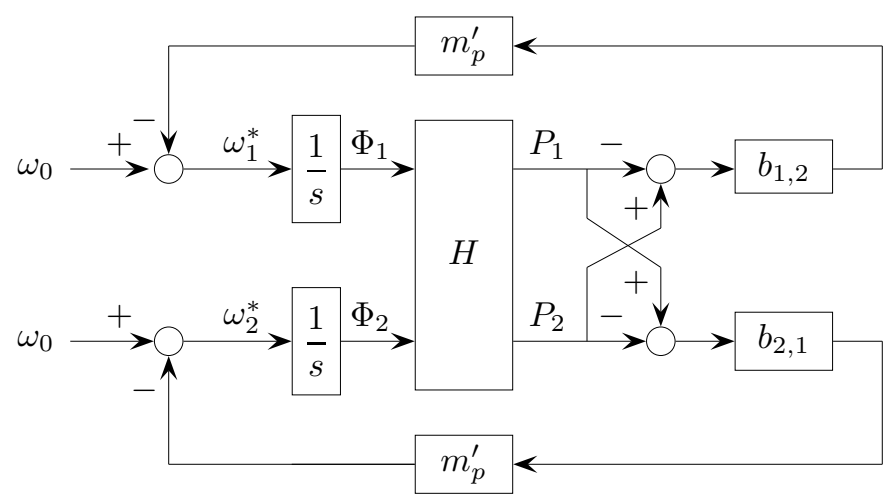

(b) Droop-free policy (9)

Fig. 3: Block diagram for the droop-based and droop-free policies for the case of two inverters.

where $m_{p}^{\prime}$ is the control gain and $b_{i, j}$ are communication weights that are also design parameters that specify the connectivity structure of the MG. Both droop-based (8) and droopfree (9) policies are illustrated as block diagrams in Figure 3 for the case of two inverters. For each policy, the top loop corresponds to one inverter and the bottom loop corresponds to the other inverter. In can be observed that in the droop-based policies the corrective terms $\delta_{1}$ and $\delta_{2}$ have not be specified yet. For the droop-free policy, it can be observed the exchange of active power $P_{1}$ and $P_{2}$ that takes place among the two inverters.

Table I summarizes the evaluated policies that are introduced next, being some of them also illustrated as block diagrams in Figure 4 for the case of two inverters. The first policy, termed as Droop-only control, is the standard droop method locally implemented at each inverter (with $\delta_{i}(t)=0$ in (8), sub-figure 3a). Although for this policy the effects of drifts were analyzed in [27], it is included in the analysis to serve as a baseline policy for comparative purposes. The next policy termed Local-integrals is the straightforward extension of the Droop-only to secondary control because each corrective term given in (12) is an integral controller of the local frequency error locally computed at each VSI (sub-figure 4a). 
TABLE I: MG control policies under evaluation

\begin{tabular}{cccc}
\hline \hline Policy & Control & Correction term $\left(\delta_{i}(t)=\right)$ & \\
\hline Droop-only & $(8)$ & $k_{I l} \int_{0}^{t}\left(\omega_{0}-\omega_{i}^{*}(t)\right) \mathrm{d} t$ & $(12)$ \\
Local-integrals & $(8)$ & $k_{I c} \int_{0}^{t}\left(\omega_{0}-\omega_{m}^{*}(t)\right) \mathrm{d} t$ & $(13)$ \\
Centralized & $(8)$ & $k_{I d} \int_{0}^{t}\left(\omega_{0}-\omega_{m}^{*}(t)\right) \mathrm{d} t$ \\
Decentralized & $(8)$ & $k_{I a} \int_{0}^{t}\left(\omega_{0}-\omega_{a}^{*}(t)\right) \mathrm{d} t$ \\
Averaging & $(8)$ & $k_{c} \int_{0}^{t}\left(\omega_{0}-\omega_{i}^{*}(t)\right.$ \\
& & $\left.+\alpha\left(\delta_{a}(t)-\delta_{i}(t)\right)\right) \mathrm{d} t$
\end{tabular}

A preliminary drift analysis for this policy is in [28].

The next four policies implement different secondary control policies with specific local integral controls ((13)-(16) in Table I) with a common denominator: they rely on a communication infrastructure linking VSI for the exchange of control data (the communication paths are marked with dashed blue lines in Sub-figures 4b-4e). In the policy termed as Centralized the corrective term (13) that is used at each VSI, namely $\delta_{m}(t)$, is again an integral controller of the frequency error computed in a central control unit, often called MGCC (MG central controller) [11], using its set-point frequency $\omega_{m}^{*}(t)$. The $\delta_{m}(t)$ term is then sent to each VSI for droop control (8) (sub-figure 4b) In the Decentralized control policy (see the overview given e.g. in [18]) the frequency error in (14) is calculated at each VSI using the received set-point frequency computed and sent by the MGCC, $\omega_{m}^{*}(t)$ (sub-figure 4c). In the Averaging control policy (see [24] or [19] for DC MG) the corrective term (15) is calculated at each VSI using the averaged set-point frequency computed as

$$
\omega_{a}^{*}(t)=\frac{1}{n} \sum_{j=1}^{n} \omega_{j}^{*}(t)
$$

where $n-1$ frequencies have been received from the others MG VSIs (sub-figure 4d). The Consensus control policy (see for example [20], sub-figure 4e) is characterized by a correction term (16) that considers two errors, the frequency one, $\omega_{0}-\omega_{i}^{*}(t)$, and the error on the correction term, $\delta_{a}(t)-\delta_{i}(t)$, where

$$
\delta_{a}(t)=\frac{1}{n} \sum_{j=1}^{n} \delta_{j}(t)
$$

The last policy listed in Table I, termed using the original name [25], Droop-free, does not relay on the droop method and its operation specified in (9) directly ensures frequency regulation and power sharing. Its application requires communications because all VSI must send their measured $P_{i}(t)$ (sub-figure 3b).

\section{B. MG control policies in terms of local times}

Table II summarizes each MG control policy previously given in Table I when they are expressed in local time coordinates. For the Droop-only policy given in (8) and the Local-integrals policy given by (8) and (12), their expression in terms of each VSI local time $t_{i}$ is straightforward and is given in (17) and (18), respectively.

For the Centralized policy specified by (8) and (13), only the MGCC controller computes the integral term. Therefore, its set-point frequency $\omega_{m}^{*}\left(t_{m}\right)$ will be computed depending only on its local time $t_{m}$, as indicated in (19). For the rest of VSIs, the computation of $\omega_{i}^{*}\left(t_{i}\right)$ will depend only on local times $t_{i}$ because the integral term is just a "quantity" received from the MGCC, as indicated in (20).

The Decentralized policy given in (8) and (14) differs from the Centralized in the fact that integrals are computed locally in terms of local times $t_{i}$, with a measure given by the MGCC in terms of its own time, $t_{m}$. Therefore, the MGCC set-point frequency $\omega_{m}^{*}\left(t_{m}\right)$ is computed like in the previous policy, as indicated in (21). For the rest of VSIs, the computation of the local frequency set-points $\omega_{i}^{*}\left(t_{i}\right)$ will depend on local times $t_{i}$ and on the MGCC measure expressed in terms of $t_{m}$. But knowing the relation of $t_{m}$ and $t_{i}$ with the global time $t$ given in (4), it is possible to express $t_{m}$ in terms of $t_{i}$ as in

$$
t_{i}=d_{i} t, t_{m}=d_{m} t \quad \rightarrow \quad t_{m}=\frac{d_{m}}{d_{i}} t_{i}
$$

and therefore the computation of $\omega_{i}^{*}\left(t_{i}\right)$ can be expressed only in terms of local times $t_{i}$ as indicated in (22) in Table II.

In the Averaging policy given in (8) and (15) the setpoint frequency $\omega_{i}^{*}\left(t_{i}\right)$ calculation depends on the averaged frequencies (10) computed at the $i^{t h}$ VSI using the received set-point frequencies $\omega_{j}^{*}\left(t_{j}\right)$ sent by the others $j^{t h}$ VSIs (and computed with their local times $t_{j}$ ). Using the same procedure than in the previous case, the others $j^{t h}$ VSIs local times $t_{j}$ can be written in terms of the local time $t_{i}$ as

$$
\left.\begin{array}{c}
\omega_{a}^{*}\left(t_{i}\right)=\frac{1}{n} \sum_{j=1}^{n} \omega_{j}^{*}\left(t_{j}\right) \\
t_{i}=d_{i} t, t_{j}=d_{j} t
\end{array}\right\} \rightarrow \omega_{a}^{*}\left(t_{i}\right)=\frac{1}{n} \sum_{j=1}^{n} \omega_{j}^{*}\left(\frac{d_{j}}{d_{i}} t_{i}\right)
$$

and the computation of $\omega_{i}^{*}\left(t_{i}\right)$ can be expressed only in terms of local times $t_{i}$ as indicated in (23).

For the Consensus policy given in (8) and (16) the setpoint frequency $\omega_{i}^{*}\left(t_{i}\right)$ calculation depends on the averaged correction terms (11) computed at the $i^{\text {th }}$ VSI using the received correction terms $\delta_{j}\left(t_{j}\right)$ sent by the others $j^{\text {th }}$ VSIs (and computed with their local times $t_{j}$ ). Similar as before, the others $j^{t h}$ VSIs local times $t_{j}$ can be written in terms of the local time $t_{i}$ as

$$
\left.\begin{array}{l}
\delta_{a}\left(t_{i}\right)=\frac{1}{n} \sum_{j=1}^{n} \delta_{j}\left(t_{j}\right) \\
t_{i}=d_{i} t, t_{j}=d_{j} t
\end{array}\right\} \rightarrow \delta_{a}\left(t_{i}\right)=\frac{1}{n} \sum_{j=1}^{n} \delta_{j}\left(\frac{d_{j}}{d_{i}} t_{i}\right)
$$

and the computation of $\omega_{i}^{*}\left(t_{i}\right)$ can be expressed only in terms of local times $t_{i}$ as indicated in (24).

Note that in (26), (27) and (28) the same global time $t$ is assumed for $t_{m}, t_{i}$ and/or $t_{j}$. Hence, it is implicitly assumed that the data transfer via the communication network is instantaneous (zero latency) and deterministic (no jitter). 


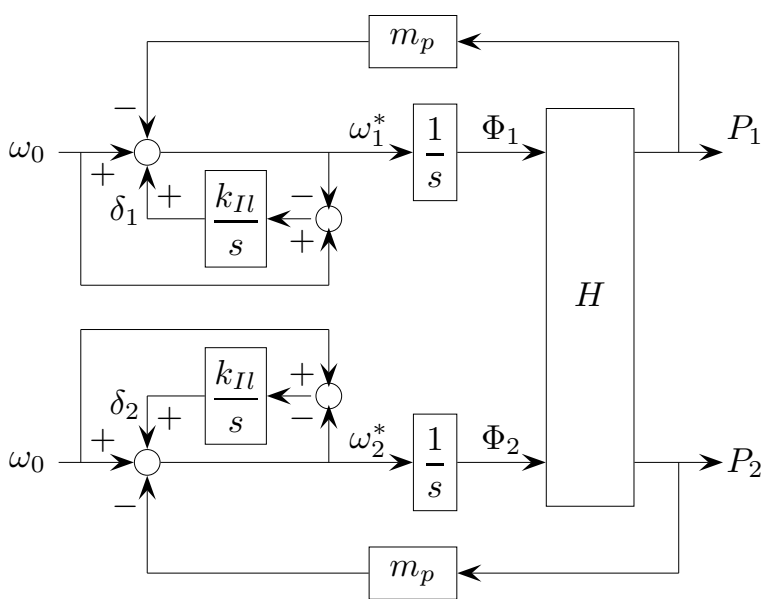

(a) Local-integrals (12)

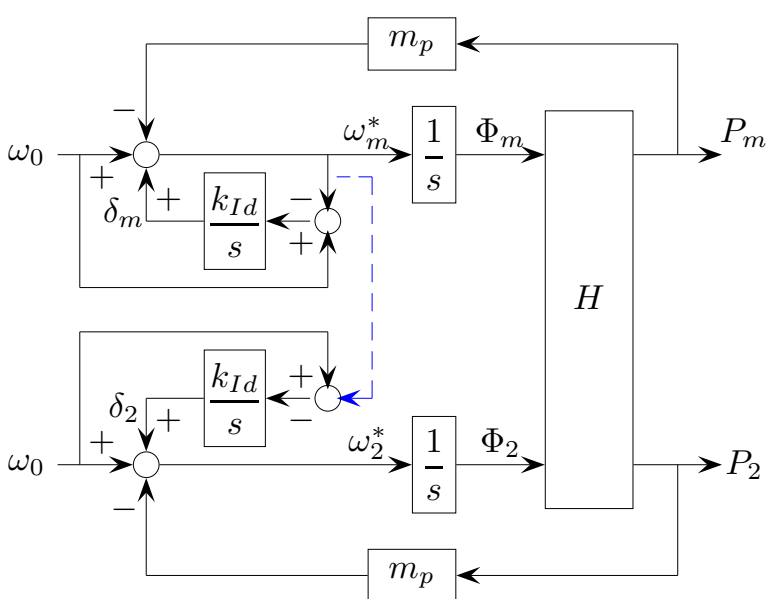

(c) Decentralized (14)

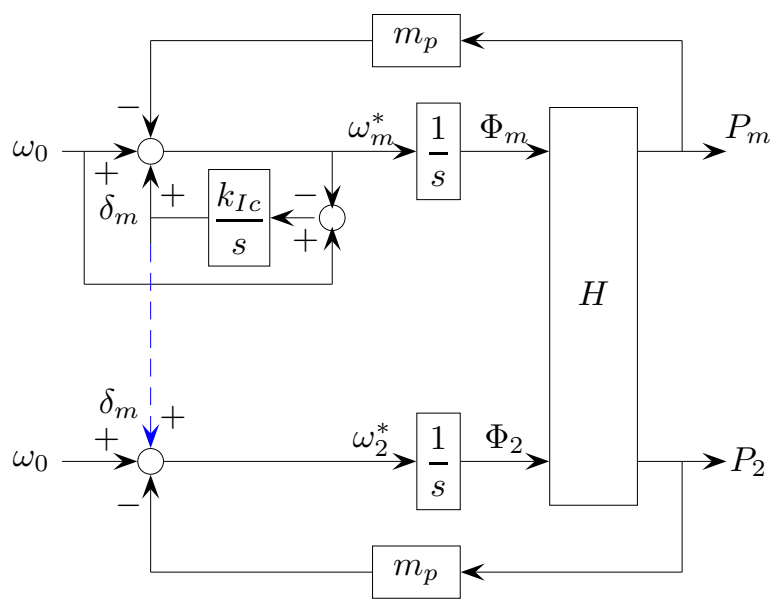

(b) Centralized (13)

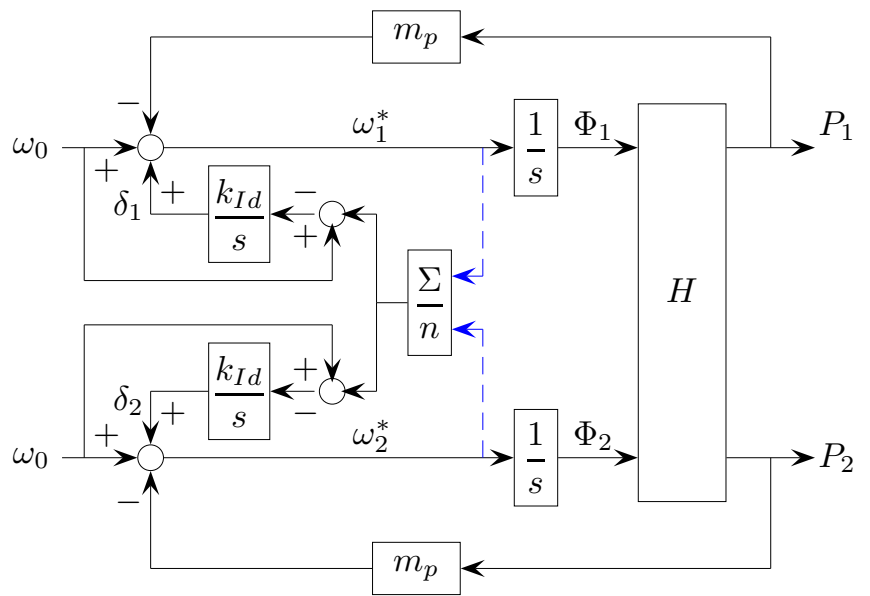

(d) Averaging (15)

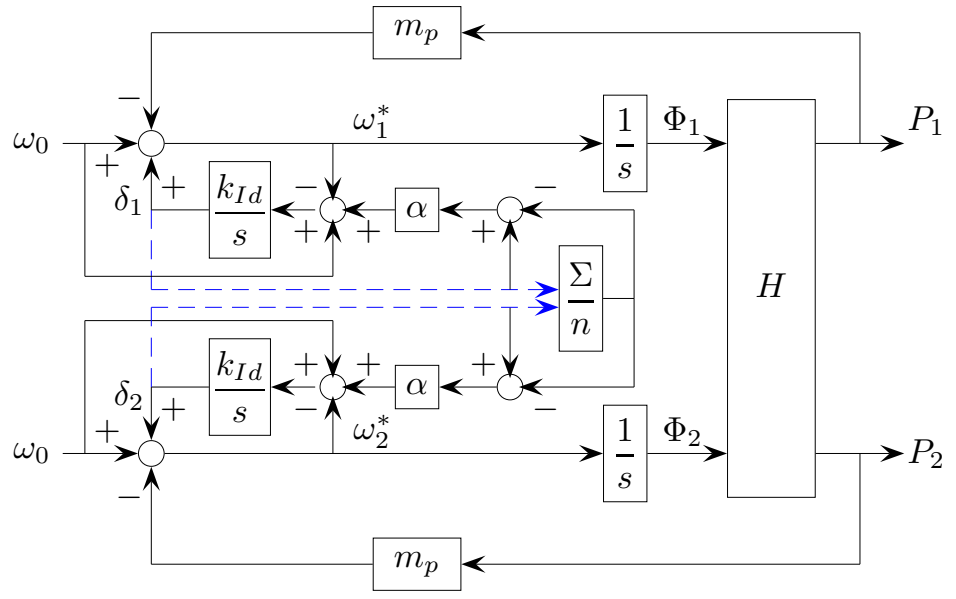

(e) Consensus (16)

Fig. 4: Block diagram for the diverse droop-based policies for the case of two inverters.

For the Droop-free policy given in (9), the expression in terms of local times is obtained straightforward by replacing $t$ by $t_{i}$, which leads to the specification given in (25). Note that for this policy, numerical integration is not required and the received active power values are just "quantities". Hence the only clock that matters at each VSI is the local one that generates $t_{i}$.

The influence of drifts can not be explicitly observed in some of the expressions of the policies shown in Table II. However, since they are expressed in terms of local times, 
TABLE II: MG control policies in terms of local times

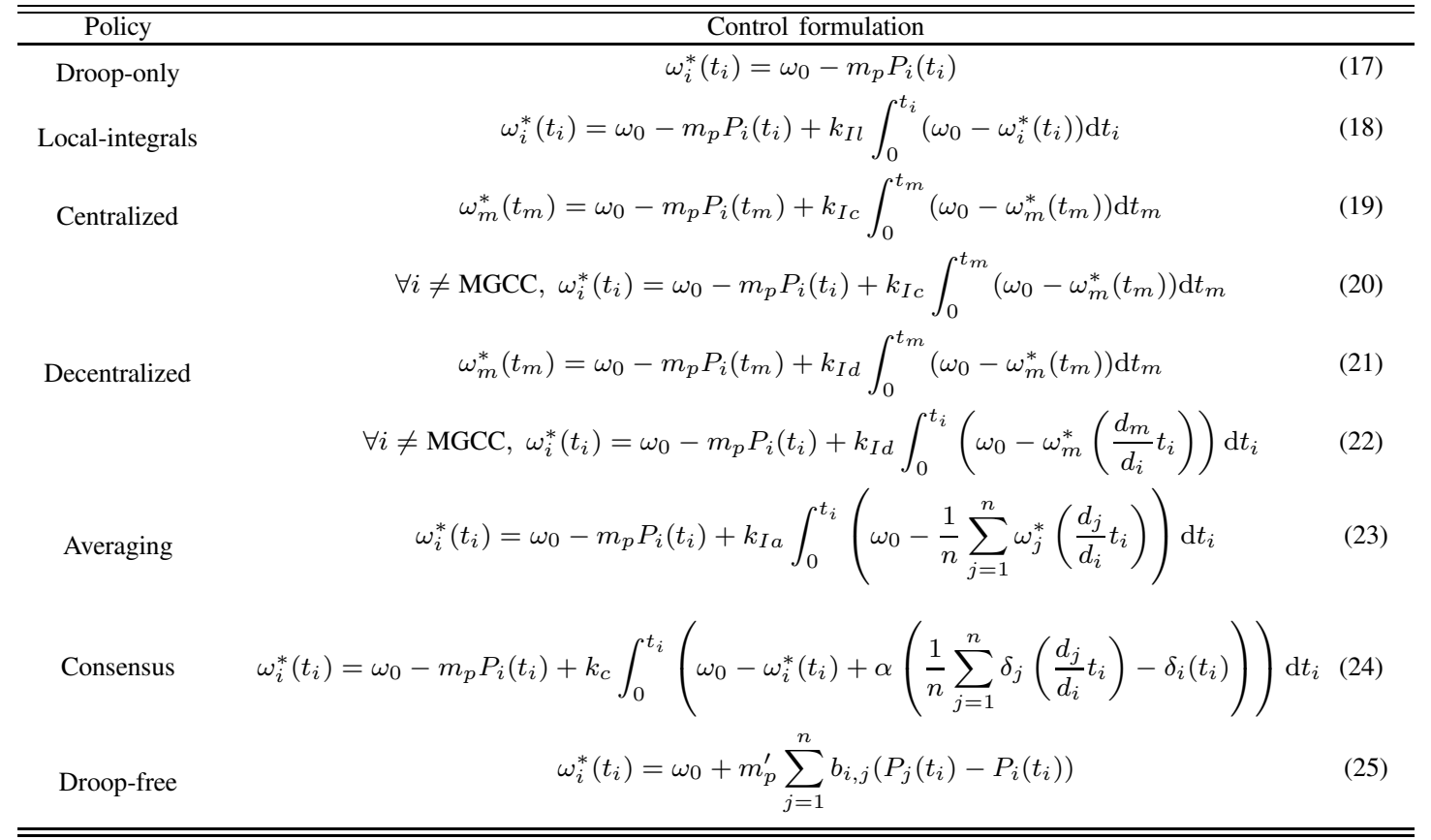

using the local time definition (4), it is straightforward to make drifts $d_{i}$ to explicitly appear in those expressions. This could help understanding the part of each policy that would be affected by the clock drift. However, it would not reveal the impact of drifts in each policy steady-state performance, which is presented next.

\section{IMPACT OF CLOCK DRIFTS: STEADY-STATE ANALYSIS}

In the previous section, the MG control policies for frequency regulation and active power sharing were characterized in terms of VSI local times. The question that arises is to assess whether both control goals can still be accomplished in the presence of divergent clocks. This section gives the answer.

\section{A. MG steady-state behavior under clock drifts}

The analyzed MG control policies were designed to reach an equilibrium frequency. Next theorem states that for droopbased policies subject to clock drifts a MG equilibrium frequency exists if local set-point frequencies are constrained by a given form, named local equilibrium frequencies.

Theorem 1. Let $t$ and $t_{i}$ be global and local times coordinates, respectively, defined by (4). For droop-based controlled VSI (6), the local set-point frequencies at each VSI must have the following form

$$
\omega_{i}^{*}\left(t_{i}\right)=\frac{C_{i, 1}}{t_{i}}+C_{i, 2}
$$

in order to ensure an equilibrium frequency $\omega(t)$ in the $M G$, with $C_{i, 1}, C_{i, 2} \in \mathbb{R}$

Proof. Let $\omega(t)$ denote the MG frequency in terms of the global time $t$. This frequency will stabilize if

$$
\frac{\mathrm{d} \omega(t)}{\mathrm{d} t}=0
$$

and $\omega(t)$ is obtained from (3) as

$$
\omega(t)=\frac{\mathrm{d} \Phi(t)}{\mathrm{d} t}
$$

Additionally, each VSI generates a local set-point frequency $\omega_{i}^{*}\left(t_{i}\right)$ in local time coordinates (see eq. (17) to (24)) that originates from the droop control (8). And with this frequency a local phase $\Phi_{i}\left(t_{i}\right)$ is generated at each VSI that should obey (3). However, considering that standard implementations of the droop method in (8) assume that the variation in active power is slow enough to consider the active power constant, the generated local phase is

$$
\Phi_{i}(t)=\int\left(\omega_{0}-m_{p} P_{i}(t)\right) \mathrm{d} t \simeq\left(\omega_{0}-m_{p} P_{i}(t)\right) t=\omega_{i}^{*}(t) t
$$

In order to obtain the MG frequency (31) in global time coordinates as a function of local times, the variation of a local phase with respect to global time can be expressed as

$$
\omega(t)=\frac{\mathrm{d} \Phi_{i}\left(t_{i}\right)}{\mathrm{d} t}
$$

By deriving the local time $t_{i}$, defined in (4), with respect to the global time $t$, it is obtained

$$
\frac{\mathrm{d} t_{i}}{\mathrm{~d} t}=d_{i} \frac{\mathrm{d} t}{\mathrm{~d} t} \quad \rightarrow \quad \mathrm{d} t=\frac{\mathrm{d} t_{i}}{d_{i}}
$$

that introduced in (33) gives

$$
\omega(t)=\frac{\mathrm{d} \Phi\left(t_{i}\right)}{\mathrm{d} t_{i}} d_{i}
$$

Then, by replacing the droop phase (32) into (35), the MG frequency in global time coordinates as a function of local times $t_{i}$ is written as

$$
\omega(t)=\frac{\mathrm{d}\left(\omega_{i}^{*}\left(t_{i}\right) t_{i}\right)}{\mathrm{d} t_{i}} d_{i}=\left[\frac{\mathrm{d} \omega_{i}^{*}\left(t_{i}\right)}{\mathrm{d} t_{i}} t_{i}+\omega_{i}^{*}\left(t_{i}\right)\right] d_{i}
$$


The condition (30) for the equilibrium frequency using (36) can be rewritten as

$$
\left[\frac{\mathrm{d}^{2} \omega_{i}^{*}\left(t_{i}\right)}{\mathrm{d} t_{i}^{2}} t_{i}+2 \frac{\mathrm{d} \omega_{i}^{*}\left(t_{i}\right)}{\mathrm{d} t_{i}}\right] d_{i}^{2}=0
$$

which is a linear homogeneous ordinary differential equation with variable coefficients known as the Cauchy-Euler equation with solution given by (29).

It is interesting to note that when time progresses, the local equilibrium frequencies given by (29) evolve to

$$
\lim _{t_{i} \rightarrow+\infty} \omega_{i}^{*}\left(t_{i}\right)=\lim _{t_{i} \rightarrow+\infty}\left(\frac{C_{i, 1}}{t_{i}}+C_{i, 2}\right)=C_{i, 2}
$$

The subsequent corollary summarizes this result.

Corollary 1. An equilibrium frequency in the MG exists if each local set-point frequency settles to a constant value when time tends to infinity.

It is interesting to observe two properties of the local equilibrium frequencies (29). First, their derivative is zero when time tends to infinity. This can be observed from their definition in (29) or from (38), and it is summarized next.

Property 1. For the local equilibrium frequencies given in (29) it holds that

$$
\lim _{t_{i} \rightarrow+\infty} \frac{\mathrm{d} \omega_{i}^{*}\left(t_{i}\right)}{\mathrm{d} t_{i}}=0
$$

The second property states that when time tends to infinity, the MG frequency in global time coordinates $\omega(\infty)$ can be directly expressed as a function of the local set-point equilibrium frequency $\omega_{i}^{*}(\infty)$ and drift rate $d_{i}$. Applying limits at the two sides of eq. (36), and using Property 1, it holds that

$$
\begin{aligned}
\omega(\infty)=\lim _{t \rightarrow+\infty} \omega(t) & =\lim _{t_{i} \rightarrow+\infty}\left[\frac{\mathrm{d} \omega_{i}^{*}\left(t_{i}\right)}{\mathrm{d} t_{i}} t_{i}+\omega_{i}^{*}\left(t_{i}\right)\right] d_{i} \\
& =\omega_{i}^{*}(\infty) d_{i}
\end{aligned}
$$

and the following property can be announced

Property 2. For the equilibrium $M G$ frequency expressed in global time coordinates $\omega(t)$ and the local equilibrium frequencies $\omega_{i}^{*}\left(t_{i}\right)$ given in (29) expressed in local time coordinates, when time tends toward infinity, they are related by

$$
\omega(\infty)=\omega_{i}^{*}(\infty) d_{i}
$$

where $d_{i}$ is the local drift rate (4).

Two more properties are announced. The first one follows from Property 2 and the second one is the power balance equation assuming negligible power losses.

Property 3. If a $M G$ equilibrium frequency $\omega(\infty)$ exists, then local equilibrium frequencies for two VSI are related by

$$
\omega_{i}^{*}(\infty) d_{i}=\omega_{j}^{*}(\infty) d_{j}
$$

where $d_{i}$ and $d_{j}$ are local drift rates (4).
Property 4. The steady-state active power $P_{i}(\infty)$ delivered by all inverters meets the MG load demand, i.e.

$$
\sum_{i=1}^{n} P_{i}(\infty)=P_{T}
$$

where $P_{T}$ is the power demanded by the MG load at $\omega(\infty)$.

Theorem 1 affirms that drifts do not affect the stability of the MG frequency in the sense that frequency equilibrium points still exist. The challenge that arises now is to determine them.

\section{B. Frequency and active power equilibrium points}

This section presents the expressions of the local equilibrium frequencies (29) and the steady-state active powers for droop-based policies (17)-(24) using the properties 1, 2, 3, and 4 that they exhibit. For the droop-free policy (25), the derivation of the steady-state properties follows a similar procedure. All results are summarized in Table III. Appendix A provides the details for deriving all the expressions shown in the table.

The steady-state values for frequencies and active powers shown in Table III analytically reveal the impact that clock drifts have in the accomplishment of the frequency regulation and power sharing control goals. The first observation refers to the existence of solutions. For all policies an equilibrium frequency exists, eqs. (45), (47), (49), (51), (53), (55), and (57), while it does not always exist an equilibrium point for the active power. In the Local-integrals policy the active power does not settle (46) and the system becomes unstable. For the rest of policies equations (44), (48), (50), (52), (54), and (56) give the active power equilibrium points.

Looking at the active power (middle column of Table III), and except for the Local-integrals, the rest of policies can be classified into two categories according to the impact of the underlying communication paradigm that they use. For those using a master/slave scheme (Centralized and Decentralized), the steady-state power value computation is different for the master $\left(d_{i}=d_{m}\right)$ and for the slaves $\left(d_{i} \neq d_{m}\right)$. This asymmetry disappears for the case of the Droop-only because it does not use communications, and for the Averaging, Consensus, and Droop-free policies that use a multi-master (assumed to be an all-to-all) communication scheme. Regarding to the size of the steady-state power sharing error, it can be observed that avoiding large errors in power sharing requires setting a high value for the droop gain $m_{p}$ (or droop-free gain $m_{p}^{\prime}$ ). This poses a conflict with the well accepted design paradigm that establishes that $m_{p}$ cannot be chosen arbitrarily large because the primary droop control may become unstable [26].

Looking at the equilibrium frequencies (right column of Table III), none of the policies is able to achieve a local setpoint frequency $\omega_{i}^{*}(\infty)$ matching the desired one $\omega_{0}$ due to the presence of clock drifts. Except for the Droop-only, that does not regulate frequency, in the rest of policies, the size of the frequency deviation will depend on the drift rates coefficients, which in general can be considered very closed to 1 and similar between them. Therefore, the frequency deviations given in (47)-(57) are expected to be very small. 
TABLE III: Steady-state active power and frequencies

\begin{tabular}{|c|c|c|c|c|c|c|c|}
\hline Policy & $\overline{P P_{i}(\infty)}$ & & & & $\overline{\omega_{i}^{*}(\infty)}$ & & \\
\hline Droop-only & $\frac{P_{T}}{\sum_{j=1}^{n} \frac{d_{i}}{d_{j}}}+\frac{\omega_{0}}{m_{p}}\left(1-\frac{n}{\sum_{j=1}^{n} \frac{d_{i}}{d_{j}}}\right)$ & (44) & $\omega_{0}-m_{p}$ & $\frac{P_{T}}{\sum_{j=1}^{n} \frac{d_{i}}{d_{j}}}$ & $+\frac{\omega_{0}}{m_{p}}$ & $1-\frac{n}{\sum_{j=1}^{n} \frac{d_{i}}{d_{j}}}$ & (45) \\
\hline Local-integrals & $\pm \infty$ & (46) & & & $\frac{n \omega_{0}}{\sum_{j=1}^{n} \frac{d_{i}}{d_{j}}}$ & & (47) \\
\hline Centralized & $\frac{P_{T}}{n}+\frac{\omega_{0} d_{m}}{m_{p}}\left(1-\frac{\sum_{j=1}^{n} \frac{d_{j}}{d_{m}}}{n}\right)-\frac{\omega_{0}\left(d_{m}-d_{i}\right)}{m_{p} d_{i}}$ & (48) & & & $\frac{\omega_{0}}{\frac{d_{i}}{d_{m}}}$ & & (49) \\
\hline Decentralized & $\frac{P_{T}}{n}+\frac{\omega_{0} d_{m}}{m_{p}}\left(1-\frac{\sum_{j=1}^{n} \frac{d_{j}}{d_{m}}}{n}\right)-\frac{\omega_{0}\left(d_{m}-d_{i}\right)}{m_{p} d_{i}}$ & (50) & & & $\frac{\omega_{0}}{\frac{d_{i}}{d_{m}}}$ & & (51) \\
\hline Averaging & $\frac{P_{T}}{n}+\frac{\omega_{0}}{m_{p}}\left(1-\frac{n}{\sum_{j=1}^{n} \frac{d_{i}}{d_{j}}}\right)$ & (52) & & & $\frac{n \omega_{0}}{\sum_{j=1}^{n} \frac{d_{i}}{d_{j}}}$ & & (53) \\
\hline Consensus & $\frac{P_{T}}{n}+\frac{\omega_{0}(\alpha+1)}{\alpha m_{p}}\left(1-\frac{n}{\sum_{j=1}^{n} \frac{d_{i}}{d_{j}}}\right)$ & (54) & & & $\frac{n \omega_{0}}{\sum_{j=1}^{n} \frac{d_{i}}{d_{j}}}$ & & (55) \\
\hline Droop-free & $\frac{P_{T}}{n}+\frac{\omega_{0}}{m_{p}^{\prime}}\left(1-\frac{n}{\sum_{j=1}^{n} \frac{d_{i}}{d_{j}}}\right)$ & (56) & & & $\frac{n \omega_{0}}{\sum_{j=1}^{n} \frac{d_{i}}{d_{j}}}$ & & (57) \\
\hline
\end{tabular}

\section{IMPACT OF CLOCK DRIFTS: STABILITY ANALYSIS}

The stability analysis is built upon the power flow expressions of the power exchanged among two consecutive inverters given by

$$
\begin{aligned}
P_{i j}(t)= & V_{i}^{2}(t) G_{i j}-V_{i}(t) V_{j}(t) G_{i j} \cos \left(\Phi_{i}(t)-\Phi_{j}(t)\right) \\
& +V_{i}(t) V_{j}(t) B_{i j} \sin \left(\Phi_{i}(t)-\Phi_{j}(t)\right)
\end{aligned}
$$

where the admittance between the two nodes in terms of the line resistance $R_{i j}$ and inductance $X_{i j}$ is

$$
G_{i j}+B_{i j} j=\frac{1}{R_{i j}+X_{i j} j}
$$

Lines are considered to be mainly inductive, that is

$$
R_{i j} \lll X_{i j} \quad \rightarrow \quad B_{i j} \approx \frac{1}{X_{i j}} .
$$

In addition, it is made explicit that each inverter voltage phase (3) is computed in terms of the drifting local time (4), that in Laplace notation can be expressed as

$$
\Phi_{i}(s)=d_{i} \frac{\omega_{i}^{*}(s)}{s} .
$$

Then, being $\hat{P}_{i j}(t)$ and $\hat{\omega}_{i}^{*}(t)$ small displacements from the equilibrium points of the exchanged power equation (58), the
Laplace small-signal model for $\hat{P}_{i j}(s)$ considering (59), (60) and (61) is given by

$$
\hat{P}_{i j}(s)=\frac{V_{i}(s) V_{j}(s)}{s X_{i j}}\left(d_{i} \hat{\omega}_{i}^{*}(s)-d_{j} \hat{\omega}_{j}^{*}(s)\right)
$$

By observing that

$$
\hat{P}_{i}(s)=\sum_{j=1, j \neq i}^{n} \hat{P}_{i j}(s)
$$

the exchanged power (78) can be written in matrix form as

$$
\hat{P}(s)=G(s) D \hat{\Omega}^{*}(s)
$$

where each power $\hat{P}_{i}(s)$ is expressed in terms of each frequency $\hat{\omega}_{i}^{*}(s)$, where

$$
G_{i j}(s)=\left\{\begin{array}{cc}
\sum_{j=1, j \neq i}^{n} \frac{V_{i}(s) V_{j}(s)}{s X_{i j}} & i=j \\
-\frac{V_{i}(s) V_{j}(s)}{s X_{i j}} & i \neq j
\end{array}\right.
$$

and where $D$ is a matrix with the drift rates in its diagonal. It is assumed that (80) already includes the Kron reduction that eliminates the nodes that do not produce power.

By making explicit that the nominal voltage frequency $\omega_{0}$ is local to each inverter time (4), and by applying the change of 
TABLE IV: Laplace transform of the MG control policies

\begin{tabular}{|c|c|c|c|}
\hline Policy & For the $i^{t h}$ inverter & & For all inverters (with $\hat{\Omega}_{0}[i]=\omega_{0}\left(\frac{s}{d_{i}}\right)$ ) \\
\hline Droop-only & $\hat{\omega}_{i}^{*}(s)=\frac{1}{d_{i}} \hat{\omega}_{0}\left(\frac{s}{d_{i}}\right)-m_{p} \hat{P}_{i}(s)$ & $(62)$ & $\hat{\Omega}^{*}(s)=D^{-1} \hat{\Omega}_{0}(s)-m_{p} \hat{P}(s)$ \\
\hline Local-integrals & $\begin{aligned} \hat{\omega}_{i}^{*}(s)= & \frac{1}{d_{i}} \hat{\omega}_{0}\left(\frac{s}{d_{i}}\right)-m_{p} \hat{P}_{i}(s) \\
& +d_{i} k_{I l} \frac{\frac{1}{d_{i}} \hat{\omega}_{0}\left(\frac{s}{d_{i}}\right)-\hat{\omega}_{i}^{*}(s)}{s}\end{aligned}$ & (64) & $\hat{\Omega}^{*}(s)=D^{-1} \hat{\Omega}_{0}(s)-m_{p} \hat{P}(s)+D k_{I l} \frac{D^{-1} \hat{\Omega}_{0}(s)-\hat{\Omega}^{*}(s)}{s}$ \\
\hline Centralized & $\begin{aligned} \hat{\omega}_{m}^{*}(s)= & \frac{1}{d_{m}} \hat{\omega}_{0}\left(\frac{s}{d_{m}}\right)-m_{p} \hat{P}_{m}(s) \\
& +d_{m} k_{I c} \frac{\frac{1}{d_{m}} \hat{\omega}_{0}\left(\frac{s}{d_{m}}\right)-\hat{\omega}_{m}^{*}(s)}{s} \\
\hat{\omega}_{i}^{*}(s)= & \frac{1}{d_{i}} \hat{\omega}_{0}\left(\frac{s}{d_{i}}\right)-m_{p} \hat{P}_{i}(s) \\
& +d_{m} k_{I c} \frac{\frac{1}{d_{m}} \hat{\omega}_{0}\left(\frac{s}{d_{m}}\right)-\hat{\omega}_{m}^{*}(s)}{s}\end{aligned}$ & 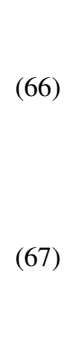 & $\hat{\Omega}^{*}(s)=D^{-1} \hat{\Omega}_{0}(s)-m_{p} \hat{P}(s)+D k_{I c} \mathbb{M}_{n} \frac{D^{-1} \hat{\Omega}_{0}(s)-\hat{\Omega}^{*}(s)}{s}$ \\
\hline Decentralized & $\begin{aligned} \hat{\omega}_{m}^{*}(s)= & \frac{1}{d_{m}} \hat{\omega}_{0}\left(\frac{s}{d_{m}}\right)-m_{p} \hat{P}_{m}(s) \\
& +d_{m} k_{I d} \frac{\frac{1}{d_{m}} \hat{\omega}_{0}\left(\frac{s}{d_{m}}\right)-\hat{\omega}_{m}^{*}(s)}{s} \\
\hat{\omega}_{i}^{*}(s)= & \frac{1}{d_{i}} \hat{\omega}_{0}\left(\frac{s}{d_{i}}\right)-m_{p} \hat{P}_{i}(s) \\
& +d_{m} k_{I d} \frac{\frac{1}{d_{m}} \hat{\omega}_{0}\left(\frac{s}{d_{m}}\right)-\hat{\omega}_{m}^{*}(s)}{s}\end{aligned}$ & 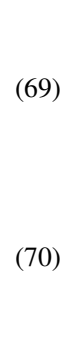 & $\hat{\Omega}^{*}(s)=D^{-1} \hat{\Omega}_{0}(s)-m_{p} \hat{P}(s)+D k_{I d} \frac{D^{-1} \hat{\Omega}_{0}(s)-\mathbb{M}_{n} \hat{\Omega}^{*}(s)}{s}$ \\
\hline Averaging & $\begin{aligned} \hat{\omega}_{i}^{*}(s)= & \frac{1}{d_{i}} \hat{\omega}_{0}\left(\frac{s}{d_{i}}\right)-m_{p} \hat{P}_{i}(s) \\
& +d_{i} k_{I a} \frac{\frac{1}{d_{i}} \hat{\omega}_{0}\left(\frac{s}{d_{i}}\right)-\hat{\omega}_{a}^{*}(s)}{s}\end{aligned}$ & (72) & $\hat{\Omega}^{*}(s)=D^{-1} \hat{\Omega}_{0}(s)-m_{p} \hat{P}(s)+D k_{I a} \frac{D^{-1} \hat{\Omega}_{0}(s)-\frac{1}{n} \mathbb{1}_{n} \hat{\Omega}^{*}(s)}{s}$ \\
\hline Consensus & $\begin{aligned} \hat{\omega}_{i}^{*}(s)= & \frac{1}{d_{i}} \hat{\omega}_{0}\left(\frac{s}{d_{i}}\right)-m_{p} \hat{P}_{i}(s) \\
& +d_{i} k_{c} \frac{\frac{1}{d_{i}} \hat{\omega}_{0}\left(\frac{s}{d_{i}}\right)-\hat{\omega}_{i}^{*}(s)+\alpha\left(\hat{\delta}_{a}(s)-\hat{\delta}\right.}{s}\end{aligned}$ & $\frac{\left.\hat{\delta}_{i}(s)\right)}{(74)}$ & $\begin{aligned} \hat{\Omega}^{*}(s)= & D^{-1} \hat{\Omega}_{0}(s)-m_{p} \hat{P}(s) \\
& +k_{c}\left[\left(D^{-1} s+\alpha\right) I-\frac{\alpha}{n} \mathbb{1}_{n}\right]^{-1}\left[D^{-1} \hat{\Omega}_{0}(s)-\hat{\Omega}^{*}(s)\right]\end{aligned}$ \\
\hline Droop-free & $\hat{\omega}_{i}^{*}(s)=\frac{1}{d_{i}} \hat{\omega}_{0}\left(\frac{s}{d_{i}}\right)+m_{p}^{\prime} \sum_{j=1}^{n} b_{i, j}\left(\hat{P}_{j}(s)-\hat{P}_{i}(s)\right)$ & (76) & $\hat{\Omega}_{i}^{*}(s)=D^{-1} \hat{\Omega}_{0}(s)+m_{p}^{\prime} \mathcal{L}_{b} \hat{P}(s)$ \\
\hline
\end{tabular}

scale property of the Laplace transform that allows expressing a function of drifted time as a scaled function of the scaled complex variable $s$, the Laplace transform of each policy in Table I is given in Table IV for each inverter (second column) and in compact form for all inverters (third column). In (68) and (71), matrix $\mathbb{M}_{n}$ denotes a $n \times n$ square matrix with all its entries being 0 except for one column of 1 s in the $m$ position, in (73) and (75) matrix $\mathbb{1}_{n}$ denotes a $n \times n$ square matrix will all its entries being 1 , and in (77) matrix $\mathcal{L}_{b}$ is the Laplacian associated to the communication graph. Moreover, policies of Table IV are expressed in Table V (second column) according to the following structural pattern

$$
\hat{\Omega}^{*}(s)=H_{1}(s) \hat{\Omega}_{0}(s)-H_{2}(s) \hat{P}(s)
$$

Given the exchanged power (80), and using the structural pattern (95), the closed-loop MG model for all policies can be

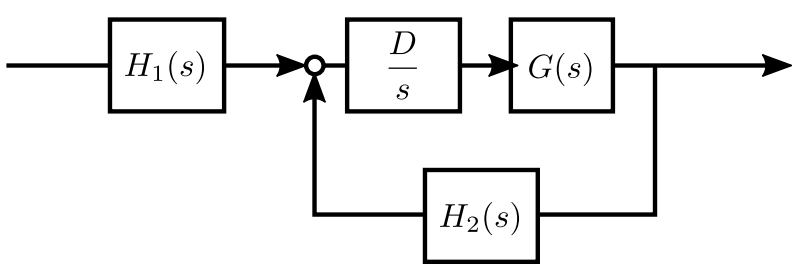

Fig. 5: Closed-loop diagram

expressed by

$$
\begin{aligned}
\hat{P}(s) & =G(s) \hat{\Omega}^{*}(s) \\
& =G(s)\left[H_{1}(s) \hat{\Omega}_{0}-H_{2}(s) \hat{P}(s)\right] \\
& =\left[I+G(s) H_{2}(s)\right]^{-1} G(s) H_{1}(s) \hat{\Omega}_{0}
\end{aligned}
$$

and schematically drawn in Figure 5. 
TABLE V: $H_{1}(s)$ and $H_{2}(s)$ for the structural pattern (95), and stability condition

\begin{tabular}{|c|c|c|c|}
\hline Policy & $H_{1}(s)$ and $H_{2}(s)$ & Stability condition & \\
\hline Droop-only & $H_{1}(s)=D^{-1}, H_{2}(s)=m_{p} I$ & $\bar{\sigma}\left(r\left[I+s^{-1} G m_{p}\right]^{-1}\left[-s^{-1} G m_{p}\right]\right)<1$ & (83) \\
\hline Local-integrals & $H_{1}(s)=D^{-1}, H_{2}(s)=m_{p} s\left[s I+D k_{I l}\right]^{-1}$ & (It does not apply) & \\
\hline Centralized & $H_{1}(s)=D^{-1}, H_{2}(s)=m_{p} s\left[s I+D k_{I c} \mathbb{M}_{n}\right]^{-1}$ & (85) $\bar{\sigma}\left(p\left[s\left(I+G m_{p}\right)+K_{I c} \mathbb{M}_{n}\right]^{-1}\left[-K_{I c} \mathbb{M}_{n}-G m_{p}\right]\right)<1$ & (86) \\
\hline Decentralized & $\begin{array}{l}H_{1}(s)=\left[s I+D k_{I d} \mathbb{M}_{n}\right]^{-1}\left[s I+D k_{I d} I\right] D^{-1} \\
H_{2}(s)=m_{p} s\left[s I+D k_{I d} \mathbb{M}_{n}\right]^{-1}\end{array}$ & $\bar{\sigma}\left(p\left[s\left(I+G m_{p}\right)+K_{I a} \mathbb{M}_{n}\right]^{-1}\left[-K_{I a} \mathbb{M}_{n}-G m_{p}\right]\right)<1$ & (88) \\
\hline Averaging & $\begin{array}{l}H_{1}(s)=\left[s I+D k_{I a} \mathbb{1}_{n}\right]^{-1}\left[s I+D k_{I a} I\right] D^{-1} \\
H_{2}(s)=m_{p} s\left[s I+D k_{I a} \mathbb{1}_{n}\right]^{-1}\end{array}$ & $\bar{\sigma}\left(p\left[s\left(I+G m_{p}\right)+K_{I d} \mathbb{1}_{n}\right]^{-1}\left[-K_{I d} \mathbb{1}_{n}-G m_{p}\right]\right)<1$ & (90) \\
\hline Consensus & $\begin{array}{l}H_{1}(s)=D^{-1} \\
H_{2}(s)=m_{p}\left[I+k_{c}\left[\left(D^{-1} s+\alpha\right) I-\frac{\alpha}{n} \mathbb{1}_{n}\right]^{-1}\right]\end{array}$ & $\begin{array}{c}{ }_{-1} \quad \bar{\sigma}\left(r s^{-1}\left(\alpha-\frac{\alpha}{n}\right) \mathbb{1}_{n}\left[I+K_{c}\left[s+s G m_{p}\right]^{-1}\left[I+\left(\alpha-\frac{\alpha}{n}\right) \mathbb{1}_{n}\right]^{-1}\right.\right. \\
\text { (91) } \\
\left.\left[I+\left(\alpha-\frac{\alpha}{n}\right) \mathbb{1}_{n}\right]^{-1}\right)<1\end{array}$ & ]$^{1}$ \\
\hline Droop-free & $H_{1}(s)=D^{-1}, H_{2}(s)=m_{p}^{\prime} \mathcal{L}_{b}$ & $\bar{\sigma}\left(r\left[s+G m_{p} \mathcal{L}_{b}\right]^{-1}\left[-G m_{p} \mathcal{L}_{b}\right]\right)<1$ & (94) \\
\hline
\end{tabular}

The closed-loop system (96) is analyzed using robust control techniques to assess the stability of the equilibrium points considering clock drifts. In particular the MG is treated as an uncertain system where the uncertainty is given by each drift $d_{i}$ (gathered in $D$ ). The structure of the uncertainty for each policy is given by the feedback path characterized by $H_{2}(s)$ and it can be modeled using an additive structured uncertainty $D=I+r \Delta$, where $I$ is the identity matrix, $r$ is the scalar value given by the drifts upper bound, and $\Delta$ is a diagonal matrix such that $\bar{\sigma}(\Delta)<1$. For convenience, $D^{-1}$ is similarly defined as $D^{-1}=I+p \Delta$, where $p$ is the scalar value which represents the maximum value of the entries of $p \Delta$. Table V (third column) shows the application of the small gain theorem for MIMO (multiple-input/multiple-output) systems that allows characterizing the stable equilibrium points of each policy as a function of the clock drifts that are encoded in terms of the uncertainty upper bounds $r$ or $p$.

The following observations can be made by looking at the stability conditions given in Table V. First of all, the stability condition for the Local-integrals policy is missing because no stable equilibrium point for the active power exists as indicated in Table III and therefore it can not be characterized the impact of the drift in the equilibrium point. Second, for the rest of policies it is expected that if the closed-loop system was designed to be stable, small deviations caused by clock drifts should not alter the stability nature of the equilibrium points. Third, the Droop-only and the Droop-free policies do not include in their formulations (17) and (25) the integral of the frequency error, which avoids the impact that drifts have in the integral terms, thus leading to closed-loop formulations where drifts do not appear in the feedback path (in $H_{2}(s)$ ).

\section{EXPERIMENTAL RESULTS}

The laboratory MG follows the scheme shown in Figure 6, which is explained in detail in [35] (chapter 14). The MG is

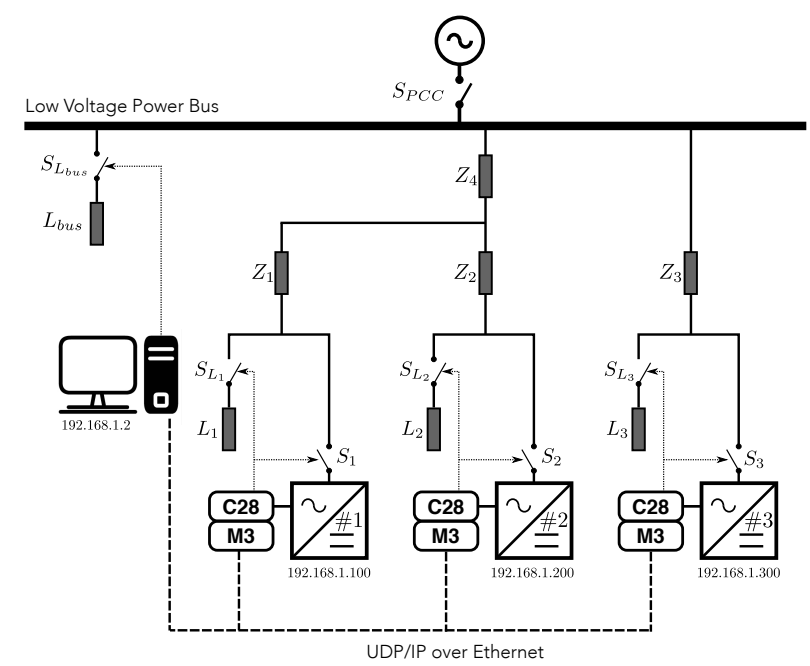

Fig. 6: Scheme for the laboratory microgrid.

composed by three VSI nodes feeding a global load, named $L_{\text {bus }}$ whose power demand is $P_{L, b u s}=1.5 \mathrm{~kW}$, that may be connected or disconnected. In all the experiments presented next, this load is permanently connected. Each power converter is driven by a dual core platform to program the control strategies shown in Table I. The chosen control platform for each VSI is a 32-bit Concerto-F28M36P63C board, composed by a C28 DSP core for power control purposes and a CortexM3 ARM core for communications. The MG uses the User Datagram Protocol (UDP)/Internet protocol (IP) protocol over an Ethernet link to allow communication among the three inverters, and with the supervisory Personal Computer (PC), where MG data is gathered for monitoring purposes. The diagram also includes line impedances modeling the parasitic elements of the cables and power transformers connected at the output of each inverter. The nominal values of the 

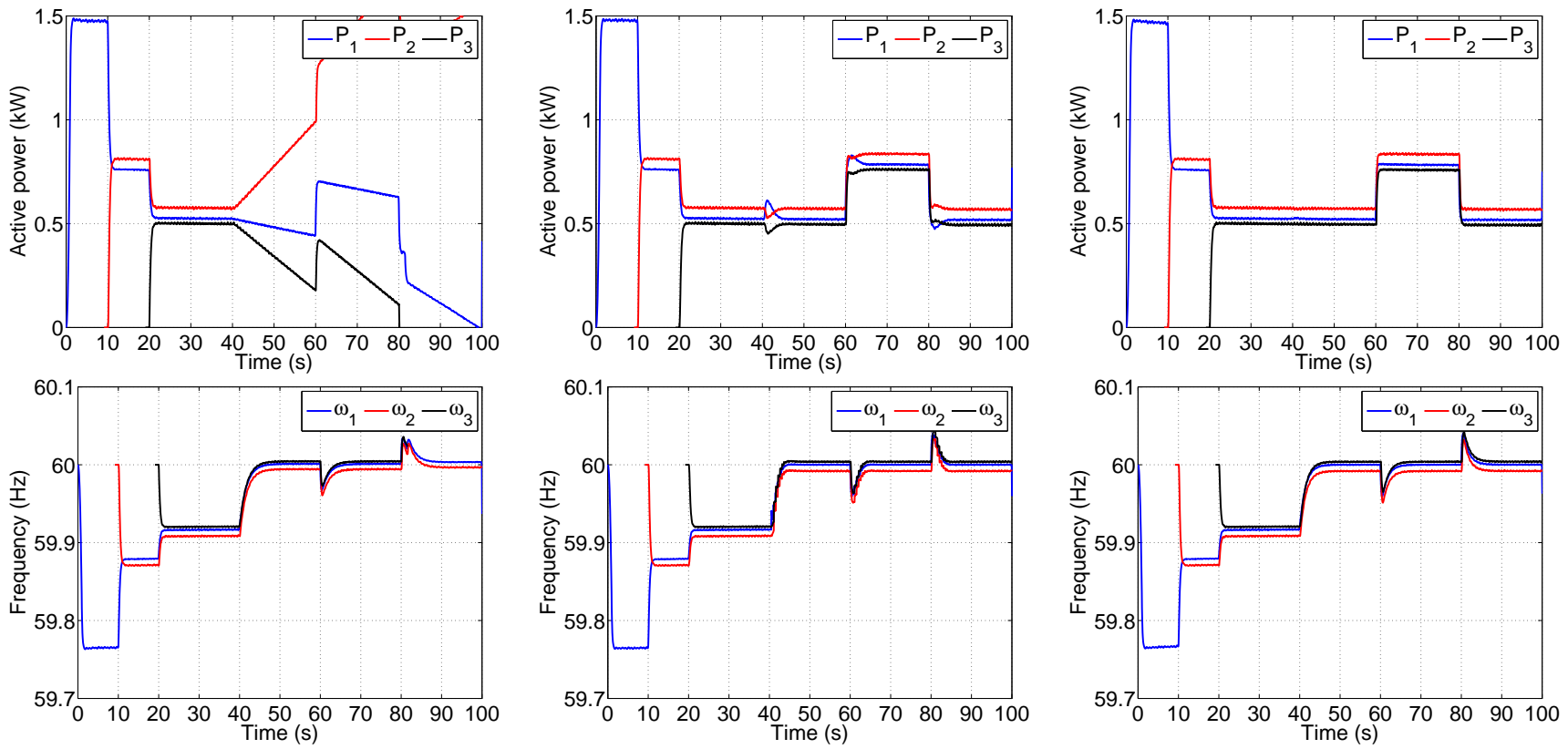

(a) Local-integrals (18)

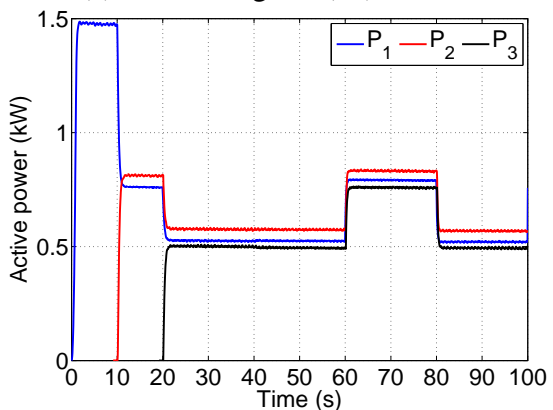

(b) Centralized (19)-(20)

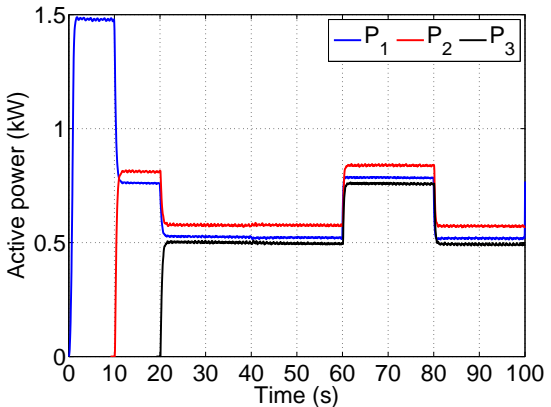

(c) Decentralized (21)-(22)
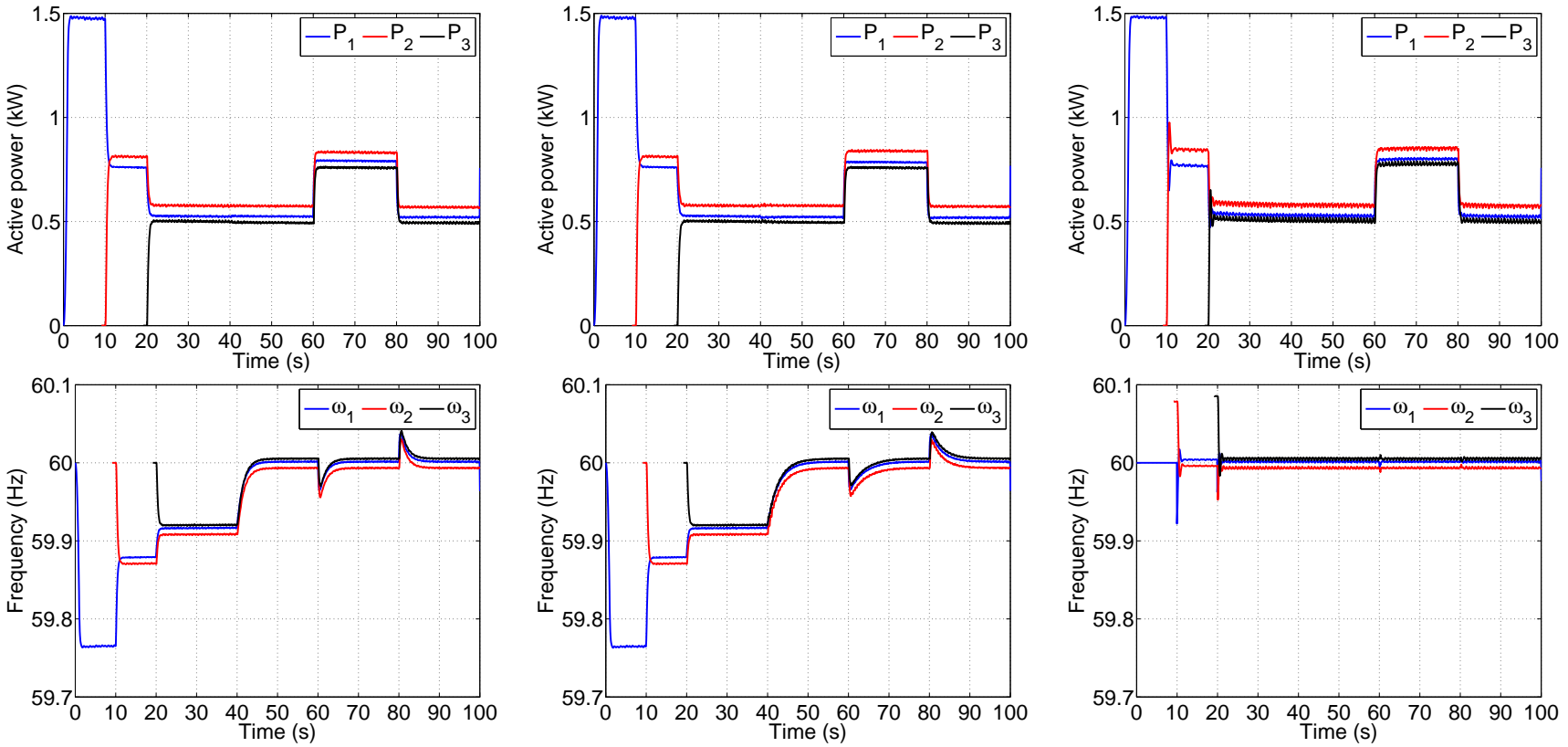

(d) Averaging (23)

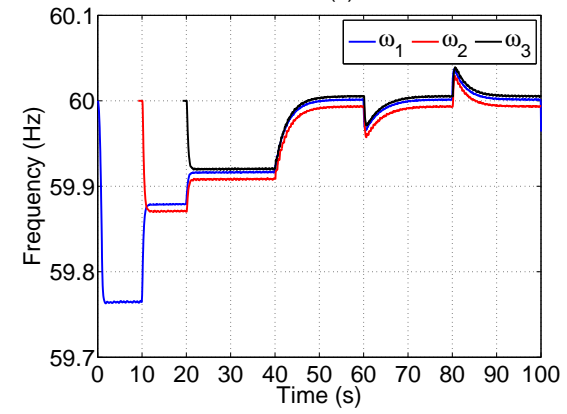

(e) Consensus (24)

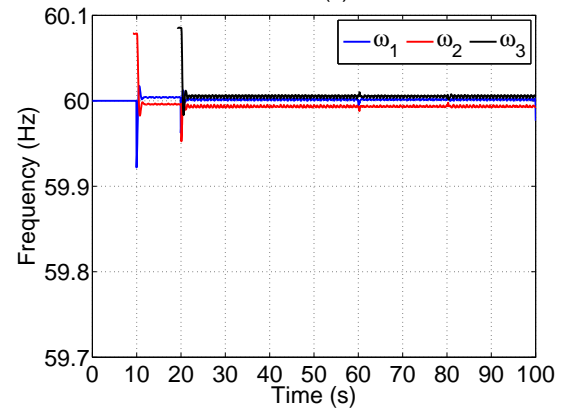

(f) Droop-free (25)

Fig. 7: Active power and frequency for the 3-nodes MG under different control policies

MG components are listed in Table VI. Each inverter has a local load $L_{i}$ with a power demand of $P_{L, i}=0.75 \mathrm{~kW}$ that allows playing with different scenarios. For the experiments presented next, $L_{1}$ has been used. The other local load remain disconnected.

The control parameters of each policy, also shown in Table VI, are kept constant whenever possible or have been defined to obtain similar dynamics among the evaluated policies. In addition, clocks drift rates have been often artificially magnified to better illustrate its effects in the steady-state values for each of the polices. The three Concerto clocks have a drift rate upper bounded by 1.00002 [37], that is, the clocks accuracy error is lower than \pm 20 parts per million (ppm). The magnified drift rates used in some of the experiments are 5 times higher, that is, with an error of $100 \mathrm{ppm}$. Note that this is however a standard number for some of the nowadays micro-controllers crystal oscillators, like those used for Ethernet communication [36]. The experiments with nonmagnified (default) drifts permit a more realistic sizing of the impact that the three Concerto clocks have on the performance of the laboratory MG. The last two parameters of Table VI refer to the transmission frequency that data is exchanged between inverters. In particular, $T_{r}$ (or $T_{r}^{\prime}$ ) is the time interval elapsed between two consecutive messages, i.e. transmission 
TABLE VI: Nominal values of the laboratory MG components

\begin{tabular}{|c|c|c|}
\hline Symbol & Description & nominal value \\
\hline$n$ & Number of inverters & 3 \\
\hline$V_{i}$ & $\begin{array}{l}\text { Inverter output voltage } \\
\text { (rms line-to-line) }\end{array}$ & $\sqrt{3} 110 \mathrm{~V}$ \\
\hline$\omega_{0}$ & Angular frequency at no load & $2 \pi 60 \mathrm{rad} / \mathrm{s}$ \\
\hline$Z_{1}$ & Line impedance 1 & $1.24 \Omega$ (0.0512p.u.), $66.15^{\circ}$ \\
\hline$Z_{2}$ & Line impedance 2 & $0.63 \Omega$ (0.0260p.u.), $37.02^{\circ}$ \\
\hline$Z_{3}$ & Line impedance 3 & $1.15 \Omega$ (0.0475p.u.), $11.32^{\circ}$ \\
\hline$Z_{4}$ & Line impedance 4 & $0.30 \Omega$ (0.0124p.u.), $90^{\circ}$ \\
\hline$P_{L, b u s}$ & Bus load power & $1.5 \mathrm{~kW}$ \\
\hline$P_{L, i}$ & Local load power & $0.75 \mathrm{~kW}$ \\
\hline$m_{p}$ & Gain of the frequency droop & $1 \mathrm{mrad} /(\mathrm{Ws})$ \\
\hline$n_{q}$ & Gain of the voltage droop & $0.5 \mathrm{mV} /(\mathrm{VAr})$ \\
\hline$L_{V}$ & Virtual impedance & $10 \mathrm{mH}$ \\
\hline$k_{P x}$ & $\begin{array}{l}\text { Integral gains } \\
\text { for policies (12)-(15) }\end{array}$ & $0.6 \mathrm{rad} / \mathrm{s}$ \\
\hline$\alpha$ & Proportional gain of consensus & 5 \\
\hline$k_{c}$ & Integral gain of consensus & $4 \mathrm{rad} / \mathrm{s}$ \\
\hline$m_{p}^{\prime}$ & Proportional gain of droop-free & $0.33 \mathrm{mrad} / \mathrm{W}$ \\
\hline$d_{1}$ & $\begin{array}{l}\text { Clock drift rate } \\
\text { of digital processor } 1\end{array}$ & 1.0000 \\
\hline$d_{2}$ & $\begin{array}{l}\text { Clock drift rate } \\
\text { of digital processor } 2\end{array}$ & 1.0001 \\
\hline$d_{3}$ & $\begin{array}{l}\text { Clock drift rate } \\
\text { of digital processor } 3\end{array}$ & 0.9999 \\
\hline$T_{s}$ & Sampling period & $100 \mu \mathrm{s}$ \\
\hline$T_{r}$ & $\begin{array}{l}\text { Transmission period } \\
\text { for policies (19)-( } 24)\end{array}$ & $0.5 \mathrm{~s}$ \\
\hline$T_{r}^{\prime}$ & $\begin{array}{l}\text { Transmission period } \\
\text { for policy }(25)\end{array}$ & $0.015 \mathrm{~s}$ \\
\hline
\end{tabular}

period, that is used for sending data in policies (19)-(24) (or policy (25)).

\section{A. General overview}

Figure 7 shows the laboratory experiments corresponding to policies (18)-(25) with magnified drift rates. For each policy, top and bottom sub-figures show the active power $P_{i}(t)$ and frequency $\omega_{i}^{*}(t)$ for the three VSI during an experiment of 100s. The experiment has the following pattern. Looking at generators, at time $t=0,10$ and 20 s the first, second and third inverter are activated, respectively. The first generator starts and fixes a MG frequency and voltage while starting to feed the bus load $\left(L_{b u s}\right)$. The activation of the second and third inverter is done by means of a phase-locked loop for synchronizing them to the MG frequency, and also start feeding the load. Looking at the control strategy, two scenarios apply. For droop-based policies (18)-(24), sub-figures 7a to 7e, up to time $t=40 \mathrm{~s}$, only droop control applies, and from $t=40 \mathrm{~s}$ to the end, the complete policy applies. The droop-free policy (25), sub-figure 7f, is always active. The first inverter adopts the master role whenever required by the policy. In addition, for all cases, at time $t=60 \mathrm{~s}$ load $L_{1}$ is connected, and it is disconnected again at time $t=80 \mathrm{~s}$.

Figure 7 does not explicitly shows the Droop-only policy (17) because it is already included in the five first policies up to time $t=40 \mathrm{~s}$. As it can be observed in subfigures 7a to 7e the local set-point frequencies (bottom subfigures) stabilize to different values that produce small error in power sharing (top sub-figures). In fact, repeating the experiment with real drifts, that is without magnified drifts, it is observed that the power sharing errors can not be appreciated
TABLE VII: Theoretical vs measured magnified drifts

\begin{tabular}{cccc}
\hline \hline & Magnified drifts & Clock drift & Real magnified drifts $d_{i}$ \\
\hline VSI $_{1}$ & 1.0000 & $-3.4535 \cdot 10^{-5}$ & 0.999965464 \\
VSI $_{2}$ & 1.0001 & $3.4185 \cdot 10^{-7}$ & 1.000100341 \\
VSI $_{3}$ & 0.9999 & $6.8259 \cdot 10^{-7}$ & 0.999900682 \\
\hline \hline
\end{tabular}

and can be considered negligible, as further analyzed in subsection VI-C, thus experimentally corroborating the claim in [27] that the droop method is robust to clock drifts.

Still looking at sub-figures $7 \mathrm{a}$ to $7 \mathrm{e}$ that correspond to the droop-based policies, a dramatic difference exists between sub-figure $7 \mathrm{a}$ that corresponds to Local-integrals and the rest of policies. As announced in [28], the (small) divergence in the local set-point frequencies provokes instability in the delivered active powers. Note that the same occurs for real drifts (as it will be shown in sub-section VI-C). For the rest of droop-based policies, sub-figures $7 \mathrm{~b}$ to $7 \mathrm{e}$, the divergency in the steady state frequencies theoretically announced in the previous section does not have a critical impact on the delivered active powers. In fact, for these policies, the dynamics of $P_{i}(t)$ slightly differ but the steady-state power sharing error is similar to the case of the droop-only scenario, which permits concluding that the performance delivered by these policies is not significantly affected by clock drifts.

The Droop-free policy shown in sub-figure $7 \mathrm{f}$ presents a different profile in active power and frequency because the policy is always active. However, similar properties with respect to the rest of policies can be observed. That is, small differences in frequency leads to small power sharing errors for the scenario of magnified drifts. Hence, the policy also performs well even in the presence of local clock drifts.

In terms of numbers, for all policies shown in Figure 7 all equilibrium frequencies lie in $60 \pm 0.01 \mathrm{~Hz}$, and all power sharing steady state values present a deviation bounded by $\pm 50 \mathrm{~W}$. Remind that this numbers are obtained for magnified drifts. Sub-section VI-C complements these numbers in the case of not-magnified drifts.

\section{B. Theoretical results versus experimental results}

This section assesses the correctness of the theoretical results for the steady-state values of frequencies and active powers with respect those obtained in the experiments. The evaluation is based on the same experimental set-up used in the previous section, with magnified drifts. It is important to highlight that on top of the magnified drifts, the real clock drifts also appear. Therefore, for an accurate assessment of the theoretical vs. the experimental results, the altered magnified drifts have been used. They have been obtained through measures. Table VII shows the theoretical magnified drifts, the clock measured ones, and the resulting drift $d_{i}$ that applies.

The assessment is shown in Table III, and the values correspond the time $t=70 \mathrm{~s}$ of each experiment. In particular, it shows, for each policy, the percentage error of each theoretical frequency $\omega_{i}^{*}(\infty)$ (equations (45), (47), (49), (51), (53), (55), 
TABLE VIII: Percentage errors for frequency and active power

\begin{tabular}{crrr}
\hline \hline & \multicolumn{3}{c}{ Frequency error \% $\left(\mathrm{x} 10^{-3}\right)$} \\
Policy & $e_{\omega, 1}$ & $e_{\omega, 2}$ & $e_{\omega, 3}$ \\
\hline Droop-only & 0.0033 & -0.0160 & 0.0120 \\
Local-integrals & -3.5000 & -5.7000 & 1.7000 \\
Centralized & 6.8000 & 6.8000 & 6.9000 \\
Decentralized & 7.0000 & 7.0000 & 7.0000 \\
Averaging & 0.0170 & 0.0089 & 0.0580 \\
Consensus & 1.3000 & 1.3000 & 1.4000 \\
Droop-free & -0.0690 & -0.0780 & 0.1500 \\
\hline \hline \multicolumn{4}{c}{ Power error \% } \\
Policy & $e_{P, 1}$ & $e_{P, 2}$ & $e_{P, 3}$ \\
\hline Droop-only & -0.0023 & 0.0100 & -0.0096 \\
Local-integrals & - & - & - \\
Centralized & 0.0078 & 0.0150 & -0.0270 \\
Decentralized & -0.0054 & -0.0210 & 0.0280 \\
Averaging & -0.4900 & -0.1800 & 0.7200 \\
Consensus & 0.0052 & -0.1000 & 0.1200 \\
Droop-free & 0.0600 & 0.0340 & -0.1000 \\
\hline \hline
\end{tabular}

and (57)) with respect to the experimental one $\omega_{i, m}^{*}(\infty)$, defined as

$$
e_{\omega, i}=100 \frac{\omega_{i}^{*}(\infty)-\omega_{i, m}^{*}(\infty)}{\omega_{i, m}^{*}(\infty)}
$$

and the percentage error of each theoretical active power $P_{i}(\infty)$ (equations (44), (46), (48), (50), (52), (54), and (56)) with respect to the experimental one $P_{i, m}(\infty)$, defined as

$$
e_{P, i}=100 \frac{P_{i}(\infty)-P_{i, m}(\infty)}{P_{i, m}(\infty)}
$$

From Table VIII it can be stated that there is a very good agreement between theoretical and experimental results.

\section{Important aspects}

This section complements the previous results by placing the attention to two important aspects.

First of all, the previous experimental results shown in Figure 7 were obtained with magnified drifts. By removing the artificial drifts, the deviations in frequency and power sharing due to the inherent (real) drifts still exist, as announced by the theoretical results summarized in Table III. But their effect is much smaller, and deviations are virtually inexistent for all the policies except for the Local-integrals that still exhibit the unstable behavior. Just for illustrative purposes, Figure 8 shows the case of the Averaging policy (to be compared with sub-figure $7 \mathrm{~d}$ ). The deviations in frequency and active power can be considered negligible.

Second, the last set of conducted experiments studies wether the availability of a clock synchronization protocol would significantly alter the obtained results when VSI are driven by drifting clocks that periodically synchronize. For this purpose, the IEEE 1588 precision time protocol (PTP) [38] has been implemented. The protocol synchronizes slave clocks to a master clock ensuring that events and time-stamps in all devices use the same time base. Rather than using specific hardware support for the PTP implementation (such as message detectors and time stamps units), the prototype implementation here applied is based on software interrupts, being the first inverter the master providing the source of time and the two other inverters the slaves synchronizing their clocks. The
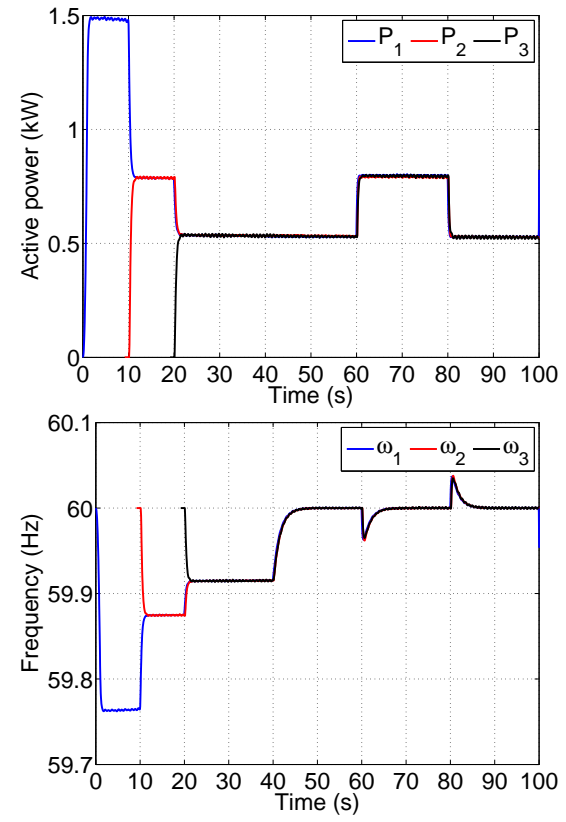

Fig. 8: The case of not-magnified clock drifts (eg. Averaging)

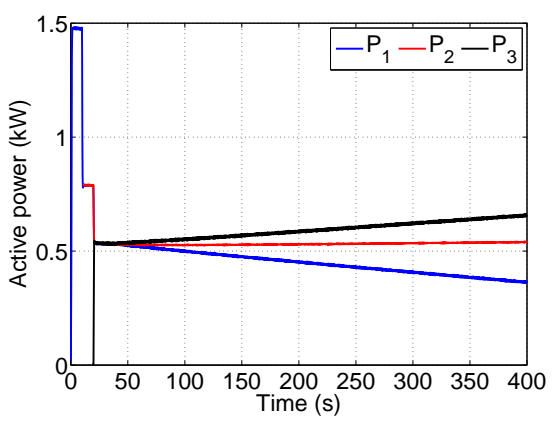

(a) Local-Integrals

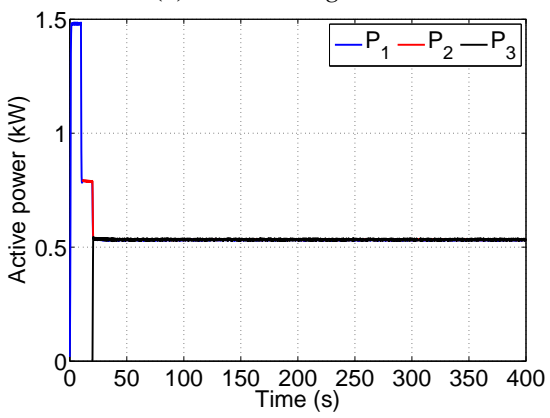

(b) Averaging

Fig. 9: The case of real drifting clocks with the IEEE1588 Precision Time Protocol for Local-integrals and Averaging policies.

execution run follows the same start-up sequence than the other experiments, but the experiment is longer and runs for 400s. The experiment is based on real default drifts, that is, no magnified drifts are artificially applied. Figure 9 summarizes the main results. Sub-figure 9a shows the active power for the case of the Local-integrals policy. As it can be observed, the application of the clock synchronization protocol does not mitigate the problem caused by clock drifts: active powers de- 
viate. Although clocks are periodically synchronized, between clock synchronization instants, the three clocks deviate again, causing a permanent local frequency error that is different in each node. Their integration in the control algorithm (18) does not stop, and as a consequence, active powers increase or decrease permanently. Sub-figure $9 \mathrm{~b}$ corresponds to the Averaging policy (taken as example within policies (19)-(25) that provide exactly the same type of results). The application of the clock synchronization protocol does not remove the announced active power steady-state deviations for these policies. But as it can be observed in the sub-figure, deviations can not be distinguished and they can be considered negligible. In summary, the application of a clock synchronization protocol does not alter the obtained results. Hence, a research challenge is the design of local control policies for frequency regulation and active power sharing being robust to clock drifts and without requiring communications for control data exchange nor for clock synchronization.

\section{CONCLUSION}

This paper has analyzed the impact that local drifting clocks have in the performance of control policies targeting frequency regulation and power sharing in VSIs-based islanded MGs. The state-of-the-art that applies has been divided into two different type of policies depending on whether they rely on the droop method. Knowing that the droop method is robust to clock drifts, the paper has analyzed the effect that local clock drifts have in the steady-state performance of those policies built on top of the droop method and those policies that do not rely on the droop method. The theoretical analysis and the experimental evidences have revealed that all the analyzed policies suffer negligible degradation due to nowadays clock drifts, except for one that makes the MG unstable.

\section{APPENDIX}

This appendix provides the theoretical derivation of the steady-state expressions for frequencies and active powers shown in Table III for each of the policies being analyzed.

1) Droop only: By using the policy definition (17) when time tends to infinity, it holds that

$$
\omega_{i}^{*}(\infty)=\omega_{0}-m_{p} P_{i}(\infty)
$$

Considering Properties 3 and 4 and (99) a system of equations is obtained whose solution is given in (44) and (45).

2) Local-integrals: The solution to the equation obtained by applying Property 1 the policy (18) is

$$
P_{i}(\infty)=\lim _{t_{i} \rightarrow+\infty} \frac{k_{I l}\left(\omega_{0}-\omega_{i}^{*}(\infty)\right)}{m_{p}} t_{i}
$$

Properties 2, 4 and (100) form a system of equations whose solution is the local equilibrium frequency given in (47). Since in general $\omega_{i}^{*}(\infty)$ are different than $\omega_{0}$, from (100) it holds that $P_{i}(\infty)$ will always increase or decrease, as indicated in (47).
3) Centralized: The solution to the equation obtained by applying Property 1 to the master policy (19) is

$$
P_{m}(\infty)=\lim _{t_{m} \rightarrow+\infty} \frac{k_{I c}\left(\omega_{0}-\omega_{m}^{*}(\infty)\right)}{m_{p}} t_{m}
$$

By applying Property 1 to the rest of VSIs (20) (and considering (26) and (101)) the resulting equation solves to

$$
P_{i}(\infty)=\frac{d_{m}}{d_{i}} P_{m}(\infty)
$$

Using Property 4 and (102) the steady-state active power given in (48) is obtained. Since $P_{i}(\infty)$ are constant, from Property 1 applied to either master or slave, it is obtained that

$$
\omega_{m}^{*}(\infty)=\omega_{0}
$$

The application of Property 2 and 3 to (103) permits deriving the local set-point frequency for any inverter, as in (49).

4) Decentralized: For this case characterized ((21) or (22)), the result for the equilibrium frequencies and active powers is the same as in the case of the centralized policy. Therefore, the mathematical derivation is omitted, but the results are given in (50) and (51). The only difference is the transient dynamics of each $\omega_{i}^{*}(t)$ because the integration term of each policy is different and is characterized by the local clock drift rate.

5) Averaging: The solution to the equation obtained by applying Property 1 to policy (23) is

$$
P_{i}(\infty)=\lim _{t_{i} \rightarrow+\infty} \frac{k_{I a}\left(\omega_{0}-\frac{1}{n} \sum_{j=1}^{n} \omega_{j}(\infty)\right)}{m_{p}} t_{i}
$$

Considering Properties 3, 4 and (104) a system of equations is obtained whose solution is given in (53). For this case, the steady-state active power stabilizes to a given value that depends on the local integral of each corrective term in (23). Assuming the same integral value for all of them, the steadystate power stabilize in (52).

6) Consensus: The solution to the equation obtained by applying Property 1 to policy (24) is

$P_{i}(\infty)=\lim _{t_{i} \rightarrow+\infty} \frac{k_{c}\left(\omega_{0}-\omega_{i}(\infty)+\frac{\alpha}{n} \sum_{j=1}^{n} \delta_{j}(\infty)-\alpha \delta_{i}(\infty)\right)}{m_{p}} t_{i}$

Considering Properties 3 and 4 and (105), and assuming that the corrective term in (24) stabilizes, i.e. $\dot{\delta}(\infty)=0$, a system of equations is obtained whose solution is given in (54) and (55).

7) Droop-free: This policy (25) does not rely on the droop method and Thereom 1 and the derived properties can not be directly applied. The phase of this policy, that applying (3) is given by

$$
\Phi_{i}(t)=\omega_{0} t+m_{p}^{\prime} \int \sum_{j=1}^{n} b_{i, j}\left(P_{j}(t)-P_{i}(t)\right) \mathrm{d} t
$$

can be written in terms of local time coordinates $t_{i}$ using the time transformation given in (26) with $t_{j}$ instead of $t_{m}$ as

$$
\Phi_{i}(t)=\omega_{0} t+\varphi_{i}(t)
$$


with

$$
\varphi_{i}\left(t_{i}\right)=m_{p}^{\prime} \int_{0}^{t_{i}} \sum_{j=1}^{n} b_{i, j}\left(P_{j}\left(\frac{d_{j}}{d_{i}} t\right)-P_{i}(t)\right) \mathrm{d} t
$$

By multiplying and dividing $\varphi_{i}(t)$ by $t_{i}$ in (107) and rearranging, the phase becomes

$$
\Phi\left(t_{i}\right)=\left(\omega_{0}+\frac{\varphi_{i}\left(t_{i}\right)}{t_{i}}\right) t_{i}
$$

which can be seen as a droop-based policy (recall (8)) with $m_{p}=0$ and a frequency correction, with set-point frequency

$$
\omega_{i}^{*}\left(t_{i}\right)=\omega_{0}+\frac{\varphi_{i}\left(t_{i}\right)}{t_{i}}
$$

The application of Property 1 to (110) simplifies to

$$
\lim _{t_{i} \rightarrow+\infty}\left[\frac{d^{2} \varphi_{i}\left(t_{i}\right)}{d t_{i}^{2}}\right]=0
$$

which alerts that the equilibrium frequency implies that

$$
\varphi_{i}\left(t_{i}\right)=\rho_{i} t_{i}
$$

with $\rho_{i} \in \mathbb{R}$ being a constant. That is, the phase (107) must always increase (or decrease) in order to have an local equilibrium set-point frequency.

Knowing that the limit as time tends to infinity of the derivative of (108) must be a constant, $\rho_{i}$, using Properties 3 and 4, and the set-point frequency definition (110), the following system of equation can be formulated

$$
\begin{aligned}
\sum_{j=1}^{n} b_{i, j}\left(P_{j}(\infty)-P_{i}(\infty)\right) & =\frac{\rho_{i}}{m_{p}^{\prime}} \\
\omega_{i}(\infty) d_{i}-\omega_{j}(\infty) d_{j} & =0 \\
\omega_{i}(\infty)-\omega_{0} & =\rho_{i} \\
\sum_{j=1}^{n} P_{i}(\infty) & =P_{T}
\end{aligned}
$$

whose solutions when $\forall i, j \quad b_{i, j}=1$ (when data exchange occurs between all the nodes and with the same weight) are (56) and (57). The solutions for the case of arbitrary weights are matrix-based algebraic expressions that deserve a deeper analysis and are out of the scope of the analysis presented in the paper.

\section{ACKNOWLEDGMENT}

This work has been supported by ELAC2014/ESE0034 from the European Union and its linked to the Spanish national project PCIN-2015-001. We also appreciate the support from the Ministry of Economy and Competitiveness of Spain under projects TEC2013-46938-R and ENE2015-64087-C2-1-R.

\section{REFERENCES}

[1] G. Coulouris, J. Dollimore, T. Kindberg, Distributed Systems: Concepts and Design, Addison Wesley Longman, Ltd., 1996

[2] C. Lenzen, T. Locher, and R. Wattenhofer, "Tight bounds for clock synchronization," in Journal of ACM, vol. 57, num. 2, Article 8, February 2010.

[3] R.H. Lasseter, "MicroGrids," in IEEE Power Engineering Society Winter Meeting, vol.1, pp.305-308 vol.1, 2002
[4] C. Buccella, C. Cecati and H. Latafat, "Digital Control of Power Converters - A Survey," in IEEE Transactions on Industrial Informatics, vol. 8, no. 3, pp. 437-447, Aug. 2012.

[5] G. Turner, J. P. Kelley, C. L. Storm, D. A. Wetz and W. J. Lee, ”Design and Active Control of a Microgrid Testbed," in IEEE Transactions on Smart Grid, vol. 6, no. 1, pp. 73-81, Jan. 2015.

[6] S. Buso and T. Caldognetto, "Rapid Prototyping of Digital Controllers for Microgrid Inverters," in IEEE Journal of Emerging and Selected Topics in Power Electronics, vol. 3, no. 2, pp. 440-450, June 2015.

[7] S. T. Cady, A. D. Domínguez-García and C. N. Hadjicostis, "A Distributed Generation Control Architecture for Islanded AC Microgrids," in IEEE Transactions on Control Systems Technology, vol. 23, no. 5, pp. 1717-1735, Sept. 2015.

[8] F. Katiraei, R. Iravani, N. Hatziargyriou, and A. Dimeas, "Microgrids management," in IEEE Power and Energy Magazine , vol. 6, no. 3, pp. 54-65, May - June 2008.

[9] D.E. Olivares, A. Mehrizi-Sani, A.H. Etemadi, C.A. Canizares, R. Iravani, M. Kazerani, A.H. Hajimiragha, O. Gomis-Bellmunt, M. Saeedifard, R. Palma-Behnke, G.A. Jimenez-Estevez and N.D. Hatziargyriou, "Trends in microgrid control," in IEEE Transactions on Smart Grid, vol. 5, no. 4, pp. 1905-1919, July 2014

[10] Y. Han, H. Li, P. Shen, E. A. A. Coelho and J. M. Guerrero, "Review of Active and Reactive Power Sharing Strategies in Hierarchical Controlled Microgrids," in IEEE Transactions on Power Electronics, vol. 32, no. 3, pp. 2427-2451, March 2017.

[11] J.M. Guerrero, J.C. Vasquez, J. Matas, J.; L.G. de Vicuña, and M. Castilla, "Hierarchical control of droop-controlled AC and DC microgrids: a general approach toward standardization," in IEEE Transactions on ndustrial Electronics, vol.58, no.1, pp.158-172, Jan. 2011

[12] A. Bidram and A. Davoudi, "Hierarchical structure of microgrids control system," in IEEE Transactions on Smart Grid, vol. 3, no. 4, pp. 19631976, Dec. 2012

[13] M.C. Chandorkar, D.M. Divan, and R. Adapa, R., "Control of parallel connected inverters in standalone AC supply systems," in EEE Transactions on Industry Applications, vol.29, no.1, pp.136-143, Jan/Feb 1993

[14] J.C. Vasquez, J.M. Guerrero, M. Savaghebi, J. Eloy-Garcia, and R. Teodorescu, "Modeling, analysis, and design of stationary-referenceframe droop-controlled parallel three-phase voltage source inverters," in IEEE Transactions on Industrial Electronics, vol.60, no.4, pp.1271-1280, April 2013

[15] J. Schiffer, R. Ortega, A. Astolfi, J. Raisch, and T. Sezi, "Conditions for stability of droop-controlled inverter-based microgrids," in Automatica, vol. 50, no. 10, pp. 2457-2469, 2014

[16] Q. Shafiee, J.M. Guerrero, and J.C. Vasquez, "Distributed secondary control for islanded microgrids - A novel approach," IEEE Transactions on Power Electronics, vol.29, no.2, pp.1018-1031, Feb. 2014

[17] W. Liu, W. Gu, W. Sheng, X. Meng, Z. Wu, W. Chen, ”Decentralized multi-agent system-based cooperative frequency control for autonomous microgrids with communication constraints," IEEE Transactions on Sustainable Energy, vol.5, no.2, pp.446-456, April 2014

[18] O. Palizban and K. Kauhaniemi, "Secondary control in AC microgrids challenges and solutions," in 2015 International Conference on Smart Cities and Green ICT Systems (SMARTGREENS), Lisbon, Portugal, 2015.

[19] P. H. Huang, P. C. Liu, W. Xiao and M. S. El Moursi, "A Novel DroopBased Average Voltage Sharing Control Strategy for DC Microgrids," in IEEE Transactions on Smart Grid, vol. 6, no. 3, pp. 1096-1106, May 2015.

[20] F. Guo, C. Wen, J. Mao, and Y.-D. Song, "Distributed secondary voltage and frequency restoration control of droop-controlled inverterbased microgrids," in IEEE Transactions on Industrial Electronics, vol. 62, no. 7, pp. 4355-4364, July 2015

[21] J. Xiao, P. Wang and L. Setyawan, "Hierarchical Control of Hybrid Energy Storage System in DC Microgrids," in IEEE Transactions on Industrial Electronics, vol. 62, no. 8, pp. 4915-4924, Aug. 2015.

[22] L.-Y. Lu and C.-C. Chu, "Consensus-based secondary frequency and voltage droop control of virtual synchronous generators for isolated AC micro-grids", in IEEE Journal on Emerging and Selected Topics in Circuits and Systems, vol. 5, no. 3, pp. 443 - 455, Sept. 2015

[23] F. Guo, C. Wen, J. Mao, J. Chen, and Y.-D. Song, "Distributed Cooperative Secondary Control for Voltage Unbalance Compensation in an Islanded Microgrid," in IEEE Transactions on Industrial Informatics, vol.11, no.5, pp.1078-1088, Oct. 2015

[24] J.W. Simpson-Porco, Q. Shafiee, F. Dorfler, J.C. Vasquez, J.M. Guerrero, and F. Bullo, "Secondary frequency and voltage control of islanded microgrids via distributed averaging," in IEEE Transactions on Industrial Electronics, vol.62, no.11, pp.7025-7038, Nov. 2015 
[25] V. Nasirian, Q. Shafiee, J.M. Guerrero, F.L. Lewis and A. Davoudi, "Droop-Free Distributed Control for AC Microgrids," in IEEE Transactions on Power Electronics, vol. 31, no. 2, pp. 1600-1617, Feb. 2016.

[26] F. Dörfler, J.W. Simpson-Porco, and F. Bullo, "Breaking the hierarchy: distributed control \& economic optimality in microgrids", in IEEE Transactions on Control of Network Systems, to appear, 2016.

[27] J. Schiffer, R. Ortega, C. A. Hans and J. Raisch, "Droop-controlled inverter-based microgrids are robust to clock drifts," 2015 American Control Conference (ACC), Chicago, IL, 2015, pp. 2341-2346.

[28] M. Velasco, P. Martí, A. Camacho, J. Miret, M. Castilla, "Synchronization of local integral controllers for frequency restoration in islanded microgrids", 42nd Annual Conference of IEEE Industrial Electronics Society, October, 2016

[29] J. Schiffer, C. A. Hans, T. Kral, R. Ortega and J. Raisch, "Modeling, Analysis, and Experimental Validation of Clock Drift Effects in LowInertia Power Systems," in IEEE Transactions on Industrial Electronics, vol. 64, no. 7, pp. 5942-5951, July 2017.

[30] A. Krishna, C. A. Hans, J. Schiffer, J. Raisch and T. Kral, "Steady state evaluation of distributed secondary frequency control strategies for microgrids in the presence of clock drifts," in 25th Mediterranean Conference on Control and Automation (MED), 2017.

[31] R. R. Kolluri, I. Mareels, T. Alpcan, M. Brazil, J. de Hoog and D. A. Thomas, "Power Sharing in Angle Droop Controlled Microgrids," in IEEE Transactions on Power Systems, vol. 32, no. 6, pp. 4743-4751, Nov. 2017.

[32] M. Castilla, A. Camacho, P. Martí, M. Velasco and M. Moradi Ghahderijani, "Impact of Clock Drifts on Communication-free Secondary Control Schemes for Inverter-based Islanded Microgrids," in IEEE Transactions on Industrial Electronics, in press, 2017.

[33] P. Martí, M. Velasco, E.X. Martín, L.G. de Vicuña, J. Miret and M Castilla, "Performance Evaluation of Secondary Control Policies with Respect to Digital Communications Properties in Inverter-based Islanded Microgrids", in in IEEE Transactions on Smart Grid, in press, 2017.

[34] J. Schiffer, D. Zonetti, R. Ortega, A.M. Stankovic, T. Sezi, and J. Raisch. "A survey on modeling of microgrids - From fundamental physics to phasors and voltage sources," in Automatica, vol. 74, issue: C, pp. 135150, Dec. 2016.

[35] M. Castilla (ed.), Control Circuits in Power Electronics: Practical issues in design and implementation, IET, ISBN: 978-1-84919-822-6, 464pp, 2016.

[36] IEEE Standard for Ethernet, in IEEE Std 802.3-2015 (Revision of IEEE Std 802.3-2012), pp. 1-4017, 2016.

[37] Schematics F28M36x controlCARD, Texas Instruments INC, Notes 2012, ftp://ftp.ti.com/pub/dml/DMLrequest/Christy_FTP-10-30-12/ controlSUITE/development_kits/ controlCARDs/TMDSCNCD28M36_ v1_0/R1_1/F28M36x_180controlCARD_R1.1_SCH.pdf

[38] "IEEE Standard for a Precision Clock Synchronization Protocol for Networked Measurement and Control Systems," in IEEE Std 1588-2008 (Revision of IEEE Std 1588-2002), pp.1-300, July 2008.

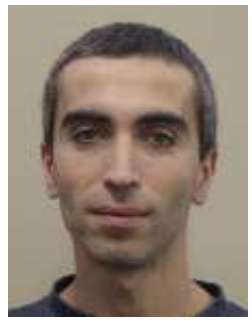

Pau Martí (M’02) received the degree in computer science and the $\mathrm{PhD}$ degree in automatic control from the Technical University of Catalonia, Barcelona, Spain, in 1996 and 2002, respectively. During 2003 he held a research fellow appointment in the Computer Science Department at the University of California at Santa Cruz, US. From 1996 to 2013, he has been an assistant professor in the Department of Automatic Control at the Technical University of Catalonia. Since 2013 he has been an Associate Professor in the Department of Automatic Control at the Technical University of Catalonia. His research interests include embedded and networked control systems, nonlinear control and microgrids.

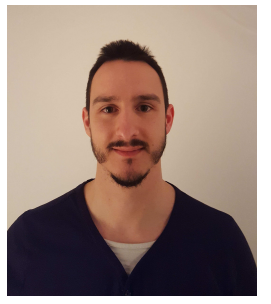

Javier Torres-Martínez received the B.S. degree in electronic engineering and the M.S. degree in automation and industrial electronics from the Technical University of Catalonia, Barcelona, Spain, in 2011 and 2013, respectively. He is currently working toward the Ph.D. degree in electronic engineering at the Technical University of Catalonia, Vilanova i la Geltr, Spain. His research interests include power electronics and control for distributed generation and microgrids.

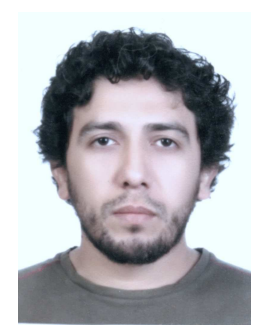

Carlos Xavier Rosero received the B.Sc. degree in automotive electronics engineering from the Army Polytechnic School, Sangolqui, Ecuador in 2008, and the M.Sc. degree in automatic control and robotics from the Technical University of Catalonia (UPC), Barcelona-Spain in 2015. He is currently working toward the Ph.D. degree in automatic control, robotics and vision from UPC. His research interests are centered on real-time control systems and microgrids with emphasis on control strategies.

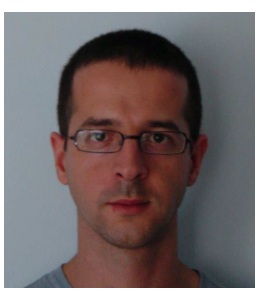

Manel Velasco graduated in maritime engineering in 1999 and received the $\mathrm{PhD}$ degree in automatic control in 2006, both from the Technical University of Catalonia, Barcelona, Spain. Since 2002, he has been an assistant professor in the Department of Automatic Control at the Technical University of Catalonia. He has been involved in research on artificial intelligence from 1999 to 2002 and, since 2000, on the impact of real-time systems on control systems. His research interests include artificial intelligence, real-time control systems, and collaborative control systems, especially on redundant controllers and multiple controllers with self-interacting systems.

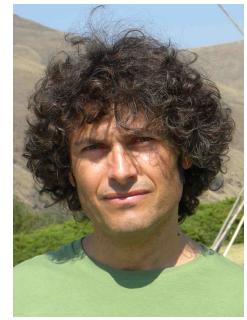

Jaume Miret (M'98) received the B.S. degree in telecommunications, M.S. degree in electronics, and $\mathrm{Ph} . \mathrm{D}$. degree in electronics from the Universitat Politecnica de Catalunya, Barcelona, Spain, in 1992, 1999, and 2005, respectively. From 1993 to 2011, he was an Assistant Professor in the Department of Electronic Engineering, Universitat Politecnica de Catalunya, Spain. Since 2011 he has been an Associate Professor in the Universitat Politecnica de Catalunya, where he teaches courses on digital design and circuit theory. His research interests include dc-to-ac converters, active power filters, and digital control.

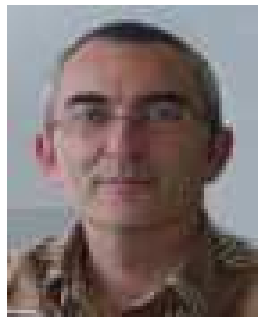

Miguel Castilla received the B.S., M.S. and Ph.D. degrees in telecommunication engineering from the Technical University of Catalonia, Barcelona, Spain, in 1988, 1995, and 1998, respectively. Since 2002, he has been an Associate Professor in the Department of Electronic Engineering, Technical University of Catalonia, where he teaches courses on analog circuits and power electronics. His research interests are in the areas of power electronics, nonlinear control, and renewable energy systems. 